\title{
Scale of gravity and the cosmological constant within a landscape
}

\author{
Michael L. Graesser ${ }^{1,2}$ and Michael P. Salem ${ }^{1}$ \\ ${ }^{1}$ California Institute of Technology, Pasadena, California 91125, USA \\ ${ }^{2}$ Department of Physics, Rutgers University, Piscataway, New Jersey 08540, USA
}

(Received 19 April 2007; published 6 August 2007)

\begin{abstract}
It is possible that the scale of gravity, parametrized by the apparent Planck mass, may obtain different values within different universes in an encompassing multiverse. We investigate the range over which the Planck mass may scan while still satisfying anthropic constraints. The window for anthropically allowed values of the Planck mass may have important consequences for landscape predictions. For example, if the likelihood to observe some value of the Planck mass is weighted by the inflationary expansion factors of the universes that contain that value, then it appears extremely unlikely to observe the value of the Planck mass that is measured within our universe. This is another example of the runaway inflation problem discussed in recent literature. We also show that the window for the Planck mass significantly weakens the anthropic constraint on the cosmological constant when both are allowed to vary over a landscape.
\end{abstract}

\section{INTRODUCTION}

Theoretical results from inflationary cosmology [1] and from string theory [2,3] motivate the possibility of an eternally inflating multiverse that is populated by an infinite number of subuniverses, each obtained via local tunneling, diffusion, and/or classical slow roll into one of a myriad of allowed metastable states. (For other motivations to consider such a landscape see, for example, Refs. [4].) In this landscape picture, each of these universes may contain different values for physical parameters, or even different particles and interactions, than those that are observed within our local universe. However, the anthropic principle [5] asserts that the physical laws that may be observed within any universe must be restricted to those that permit the evolution of observers in the first place.

Combined with the anthropic principle, the landscape picture has emerged as a plausible explanation for many striking features of our universe. In particular, it has been used to justify the "unnatural" smallness of the Higgs mass [6], the cosmological constant [7,8], and the neutrino masses [9]; to predict the size of supersymmetry breaking $[3,10]$; to describe the tilt in the spectrum of density perturbations [11] and other inflationary parameters [12]; to constrain the baryon to photon ratio [13] and the ratio of baryons to dark matter [14,15], as well as to explain some seemingly fine-tuned relationships between parameters describing the theories of quantum electrodynamics and quantum chromodynamics [16]. Nevertheless, generating precise predictions from a landscape picture faces several major challenges.

One of these challenges is to identify an anthropic criteria that is both specific and compelling. Yet even after such an anthropic condition has been defined, it is a daunting task to discern its environmental requirements, to deduce their implications for physical parameters, and then to derive the associated anthropic constraints. One may proceed by considering the variation in only one physical parameter, starting from its value within our universe. However, apparently tight constraints on any single parameter may be significantly weakened when more than one parameter is allowed to vary. This seems to be the case with both the Higgs mass [17] (see however Ref. [18]) and the cosmological constant $[19,20]$. Moreover, all anthropically allowed universes may not be connected by the continuous variation of physical parameters. For example, the seemingly viable "cold big bang" universe [21] results from independently varying several cosmological parameters to values very far from those obtained within our universe.

In addition, to calculate the expectation values of physical parameters within a landscape requires determining an appropriate measure to weight among the various possible universes [22-25]. That is, a precise anthropic criteria does not account for all of the selection effects that contribute to the probability for a particular universe to be observed. Universes may be more or less likely based on how readily they are obtained via the physical dynamics that govern the multiverse. To account for this requires a complete understanding of the multiverse, its landscape, and the governing theory. Indeed, there is a more subtle challenge underlying this program, which is to develop an appropriate and self-consistent calculus to regulate calculations involving the infinite number of infinitely expansive universes that may be contained within the multiverse [2225].

Nevertheless, a set of hypotheses to resolve these challenges may be excluded if it predicts a very low likelihood to observe a universe with some physical characteristic that our universe possesses. For example, consider a proposal that includes a specific notion of observer, a consistent calculus to determine expectation values over the multiverse, and a theory to describe the landscape including how metastable states are mapped onto universes within the multiverse. If this proposal then predicts that an exponentially small number of observers measure a cosmological 
constant at or below the value obtained in our universe, then the proposal and the specific landscape in question are probably not both correct.

We investigate the possibility that the scale of gravity may scan over the landscape. This is consistent with the results of Ref. [26], where only parameters with mass dimension were found to vary over a model of the landscape. We everywhere parametrize the scale of gravity using the (reduced) Planck mass $m_{\mathrm{P}}$. Although $m_{\mathrm{P}}$ is commonly taken to be a fixed fundamental scale, this need not be the case. For example, the multiverse may be governed by a low-energy theory with the Lagrangian,

$$
\mathcal{L}=\frac{1}{2} \sqrt{-g} M^{2} F[\phi] R+\mathcal{L}_{\phi}+\mathcal{L}^{\prime},
$$

where the fundamental mass scale is $M, R$ is the Ricci scalar, $\phi$ is the collection of fields that specify the metastable state of a universe, $\mathcal{L}_{\phi}$ is the effective Lagrangian for these fields, and $\mathcal{L}^{\prime}$ is the effective Lagrangian for matter. We assume that within each metastable state the fields $\phi$ are very massive and fixed to values $\phi \rightarrow \phi_{*}$ and are therefore nondynamical. The strength of gravity will therefore be a constant within each metastable state, determined by the effective Planck mass,

$$
m_{\mathrm{P}} \equiv \sqrt{F\left[\phi_{*}\right]} M .
$$

We also assume that within each universe $m_{\mathrm{P}}$ is fixed prior to slow-roll inflation.

Alternatively, our analysis may be viewed in the socalled Einstein frame where the scale of gravity is everywhere fixed. To accomplish this, one simply performs the conformal transformation $g_{\mu \nu} \rightarrow F^{-1}\left[\phi_{*}\right] \tilde{g}_{\mu \nu}$. Then the scale of gravity is everywhere $M$, but all other parameters with dimension mass are scaled by the factor $F\left[\phi_{*}\right]^{-1 / 2}$. Thus our analysis is equivalent to fixing the Planck mass to be the fundamental scale of physics but varying all other mass scales uniformly. Stated another way, in our analysis the frame-independent ratio of masses $m / m_{\mathrm{P}}$ scales as $F\left[\phi_{*}\right]^{-1 / 2}$, where for example $m$ may be the cutoff of the theory, the Higgs mass, or the scale of strong dynamics. This picture was previously suggested in the penultimate section of Ref. [17]. The idea that the effective value of $m_{\mathrm{P}}$ may vary across the multiverse within the context of BransDicke theory was studied in Refs. [23,27]. In addition, Ref. [28] studied a model of the form of Eq. (1) to show how inflationary dynamics can explain the hierarchy between the apparent Planck scale and the electroweak scale. As described below, our focus is different from the focus of this work.

We calculate the range over which $m_{\mathrm{P}}$ may scan while still satisfying anthropic constraints. For completeness we consider a wide range of environmental constraints. These relate to halo, galaxy, and star formation, in addition to galactic and stellar dynamics. We restrict attention to universes that possess the same particles, interactions, cou- plings, and physical scales that are observed within our universe. Note that this means the cutoff to the low-energy effective theory depends on $M$, not $m_{\mathrm{P}}$. In addition, we assume that whatever mechanisms drive inflation and provide baryogenesis are unchanged (except insofar as they depend on $m_{\mathrm{P}}$ ) across the landscape that we consider. Within the context of the model described by Eq. (1), these assumptions require the existence of a large number of states that have approximately equal particle physics parameters yet different values of $m_{\mathrm{P}}$. We do not explore the interesting case of a landscape model that permits only correlated changes in $m_{\mathrm{P}}$ and the other particle physics parameters. Note that the above restrictions are conservative in the sense that lifting them can only expand the range of allowed $m_{\mathrm{P}}$.

Many anthropic constraints relate to the formation of galaxies and depend on the spectrum of energy density fluctuations evaluated at matter-radiation equality. On any given distance scale, this spectrum has an approximately Gaussian distribution about some root mean square (rms) amplitude, and we calculate constraints as if all fluctuations have the rms amplitude. Perhaps not surprisingly, the range of $m_{\mathrm{P}}$ that is consistent with all of the anthropic constraints is rather narrow. It also depends on what models are chosen for inflation, baryogenesis, and the dark matter. As an example, if we assume that inflation is chaotic with potential $V(\varphi)=\frac{1}{2} m_{\varphi}^{2} \varphi^{2}$, that baryogenesis results from efficient leptogenesis, and assume a weakly interacting massive particle (WIMP) to be the dark matter, then anthropic considerations combine to constrain $m_{\mathrm{P}}$ to be $0.1 \lesssim \hat{m}_{\mathrm{P}} \lesssim 1.5$, where $\hat{m}_{\mathrm{P}}$ is the ratio between $m_{\mathrm{P}}$ and the value obtained within our universe, about $2.4 \times$ $10^{18} \mathrm{GeV}$.

Even a very narrow anthropic range for $m_{\mathrm{P}}$ may have significant consequences for proposals to calculate its expectation value within a landscape. In particular, it is plausible that some proposals will ultimately weight universes in part according to their inflationary expansion factor. This expansion factor depends exponentially on the number of e-folds of inflation that the universe undergoes, which in turn depends on $m_{\mathrm{P}}$. In this case the probability distribution for $m_{\mathrm{P}}$ will be peaked only where some other selection effect cancels this strong exponential dependence. This other selection effect could be a very sharp peak or boundary to the underlying landscape distribution; otherwise the effect must come from an exponentially strong anthropic dependence on $m_{\mathrm{P}}$. Yet such a strong anthropic dependence on $m_{\mathrm{P}}$ would be in conflict with the observation that $m_{\mathrm{P}}$ has even a narrow anthropic window in our universe. Thus we are forced to conclude that under these weighting schemes the observation of our universe is either extremely atypical or our value of $m_{\mathrm{P}}$ sits at some sharp peak or boundary in the underlying landscape distribution. This point is completely analogous to the " $\sigma$-problem" and " $Q$ catastrophe" identified in Refs. [29]. 
We here note that a runaway problem associated with varying the effective Planck mass during eternal inflation has already been discussed in Refs. [23,27]. These papers studied the evolution of $m_{\mathrm{P}}$ in Brans-Dicke theory when the Brans-Dicke field is allowed to be dynamical during inflation. On the other hand, we study the case where the fields $\phi$ in Eq. (1) are very massive and therefore nondynamical. The difference between these scenarios is subtle because at some level $\phi$ must be dynamical in order for the landscape to be populated within the multiverse. Our approach is to assume that the fields $\phi$ are only dynamical at the very high energies that dominate the dynamics of the multiverse. At these energies the dynamics of $\phi$ could be described as in Refs. [23,27] or they could be described by different effects. We simply treat these dynamics as unknown except to assume that the $\phi$ are fixed prior to the slow-roll inflation that eventually reheats into each of the anthropically favorable low-energy universes such as our own.

We also consider the anthropic window for the cosmological constant $\Lambda$ when both $\Lambda$ and $m_{\mathrm{P}}$ are allowed to (independently) scan over the landscape. Even when the allowed range for $m_{\mathrm{P}}$ is relatively narrow, it still allows for a significant broadening of the allowed range for $\Lambda$. To see this, note that $\Lambda$ is constrained only by Weinberg's anthropic bound [7],

$$
\rho_{\Lambda} \lesssim \rho_{\text {eq }} \sigma_{\text {eq }}^{3} .
$$

Here $\rho_{\Lambda}$ is the energy density in cosmological constant, $\rho_{\text {eq }}$ is the matter density at matter-radiation equality, and $\sigma_{\text {eq }}$ is the typical fluctuation in matter density at equality. The broadening occurs because for WIMP dark matter, decreasing $m_{\mathrm{P}}$ significantly increases $\rho_{\mathrm{eq}}$ and for most models also significantly increases $\sigma_{\text {eq }}$. For example, if we again assume chaotic inflation with potential $V(\varphi)=$ $\frac{1}{2} m_{\varphi}^{2} \varphi^{2}$ and that baryogenesis results from efficient leptogenesis, then $\rho_{\Lambda}$ may be over a million times the value observed within our universe when $\hat{m}_{\mathrm{P}} \gtrsim 0.1$. Of course, a larger anthropic window for $\rho_{\Lambda}$ does not necessarily imply that our value of $\rho_{\Lambda}$ is less likely to be observed. We illustrate the distribution of observed values of $\rho_{\Lambda}$ with a very simplified calculation. The results of this calculation suggest that to observe the cosmological constant at or below the level obtained within our universe is very unlikely unless the landscape distribution of $m_{\mathrm{P}}$ is dominated by values very near to or larger than the value obtained within our universe.

The remainder of this paper is organized as follows. In Sec. II we calculate the range of $m_{\mathrm{P}}$ allowed by anthropic constraints in universes otherwise like ours. Constraints come from a variety of cosmological processes and we summarize our results in Sec. II H. Then in Sec. III we argue that the value of $m_{\mathrm{P}}$ that we observe is extremely unlikely if universes within the landscape are weighted by their inflationary expansion factor. In this section we also discuss some caveats to this argument. The scenario where both $m_{\mathrm{P}}$ and $\Lambda$ may vary across the landscape is discussed in Sec. IV. Finally, we draw our conclusions in Sec. V.

\section{ANTHROPIC CONSTRAINTS ON THE SCALE OF GRAVITY}

It is straightforward to organize the immediate effects of changing the scale of gravity when all other mass scales and couplings are kept fixed (this implies that the cutoff of the theory is also fixed). Then scanning the Planck mass corresponds to changing the proportionality constant between the Einstein and the stress-energy tensors,

$$
G_{\mu \nu}=m_{\mathrm{P}}^{-2} T_{\mu \nu}
$$

In a homogeneous universe this simply changes the relationship between the Hubble rate $H$, its time rate of change $\dot{H}$, and the energy $(\rho)$ and pressure $(P)$ densities,

$$
H^{2}=\frac{\rho}{3 m_{\mathrm{P}}^{2}}, \quad \dot{H}=-\frac{\rho+P}{2 m_{\mathrm{P}}^{2}} .
$$

This bears upon anthropic conditions because the Hubble rate determines when particle interactions freeze out of equilibrium. This affects the relative densities of, for example, matter to radiation and protons to neutrons.

Of course the universe is only approximately homogeneous. According to the present understanding, inhomogeneities are generated by quantum fluctuations in at least one scalar field as it exits the Hubble radius during (nearly) de Sitter expansion in the early universe. If this is the case, then the Hubble rate also affects the size of the initial inhomogeneities. At late times, these inhomogeneities reenter the Hubble radius and the scale of gravity takes on a new role. Then gravity provides a self-interaction to overdensities that may cause them to grow. Overdensities that grow too large become gravitationally bound and separate from the cosmic expansion. Within these structures, the expansion of the universe is inconsequential but the scale of gravity still determines the internal dynamics.

We analyze the anthropic significance of these effects in chronological order, beginning with the effects on inflation. We then discuss baryogenesis, big bang nucleosynthesis, matter domination, structure formation, stellar dynamics, and finally the stability of stellar systems. The anthropic constraints are displayed in Fig. 1 and summarized in Sec. II H. The complexity of this analysis, along with the many uncertainties in our understanding of various cosmological processes, make a precise determination of anthropic constraints impractical. Therefore we strive for approximations that capture the key effects of scanning $m_{\mathrm{P}}$. Usually, we determine the dominant scaling behavior of a quantity with $m_{\mathrm{P}}$ and cite a precisely determined value from our universe to determine the value in another universe. Unless otherwise stated, the values for cosmological parameters within our universe are taken from the tables in Ref. [15] (note however that we work in terms of the 

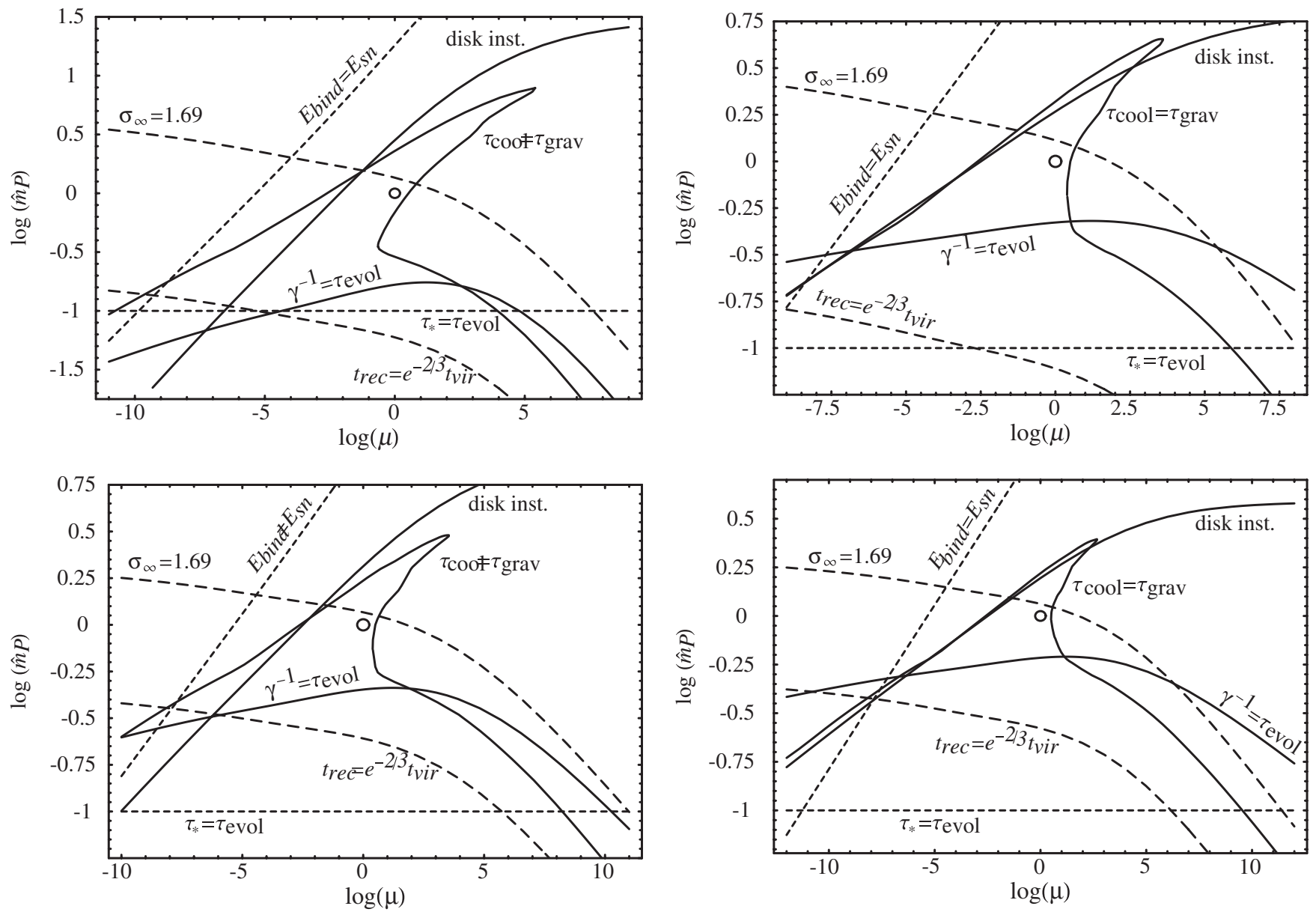

FIG. 1. Anthropic constraints on $\hat{m}_{\mathrm{P}}$, plot as a function of halo mass scale $\mu$, for $\alpha=1, \beta=0$ (top left panel); $\alpha=1, \beta=3 / 2$ (top right panel); $\alpha=3, \beta=0$ (bottom left panel); $\alpha=3, \beta=3 / 2$ (bottom right panel). The logarithms are base ten and the empty circle corresponds to the mass scale of our galaxy with $m_{\mathrm{P}}$ as observed within our universe. The region within the $\mu$ - $\hat{m}_{\mathrm{P}}$ plane that is excluded by any constraint is the region that does not include this circle. The parameters $\alpha$ and $\beta$ as well as the labels on the curves are defined in the text (see, for example, the summary of Sec. II H). Note that the galactic cooling constraint does not include the effects of molecular cooling.

reduced Planck mass). Throughout this paper we denote the ratio of a quantity to the value that it obtains within our universe using a hat, for example

$$
\hat{m}_{\mathrm{P}} \equiv \frac{m_{\mathrm{P}}}{2.4 \times 10^{18} \mathrm{GeV}} .
$$

Finally, we use units where $\hbar=c=k_{B}=1$.

\section{A. Inflation}

An early period of inflation is believed to have homogenized our universe and yet provided the seeds of cosmic structure through the generation of small density perturbations (for reviews of inflation see for example [30]). We parametrize these effects using the total number of e-folds of inflation $N$ and the Bardeen curvature perturbation $\zeta$. In principle, both $N$ and $\zeta$ are constrained by anthropic considerations. Meanwhile, for inflation to occur in the first place requires that the Hubble radius expand at a rate slower than the speed of light. This effect is parametrized by requiring that the first slow-roll parameter, $\epsilon_{I} \equiv$ $-\dot{H} / H^{2}$, is smaller than unity. Finally, at some point inflation must end and the universe must reheat to establish the initial conditions for the subsequent big bang evolution. We discuss the $m_{\mathrm{P}}$ dependence of each of these below.

\section{Satisfying slow roll for $N$ e-folds of inflation}

For inflation to occur in the first place requires that at some time $\epsilon_{I}<1$. When inflation is driven by the potential energy of a canonical scalar field $\varphi$, the first slow-roll parameter can be written

$$
\epsilon_{I} \simeq \frac{m_{\mathrm{P}}^{2}}{2}\left(\frac{V_{\varphi}}{V}\right)^{2},
$$

where $V$ is the inflaton potential and the subscript on $V_{\varphi}$ denotes differentiation with respect to $\varphi$. Although at first glance $\epsilon_{I}$ appears to increase with increasing $m_{\mathrm{P}}$, this can 
usually be compensated for by starting the inflaton $\varphi$ further up the potential. This is the case with each of the canonical inflationary models presented below. Therefore we assume that the occurrence of inflation in the first place does not significantly constrain $m_{\mathrm{P}}$.

Meanwhile, the total duration of inflation is constrained by the need to homogenize a universe large enough to allow for the formation of structure. This constraint, however, is very weak, since our observable universe appears immensely larger than is necessary to form a galaxy. Moreover, inflationary scenarios that predict the observed value of $\zeta$ typically allow for far more e-folds of inflation than are necessary to enclose our universe. Therefore we assume that the anthropic bound on $N$ does not significantly constrain $m_{\mathrm{P}}$.

\section{The curvature perturbation $\zeta$}

Anthropic constraints on the amplitude of $\zeta$ stem from primordial black hole production, structure formation, and the stability of stellar systems and are described in Secs. II D, II E, and II G. Presently we discuss the dependence $\zeta\left(m_{\mathrm{P}}\right)$ for future reference. For inflation driven by a canonical scalar $\varphi$, the curvature perturbation on a comoving scale with wave vector $k$ is

$$
\left.\zeta(k) \propto \frac{V^{3 / 2}}{m_{\mathrm{P}}^{3} V_{\varphi}}\right|_{k=a H},
$$

where $V$ and $V_{\varphi}$ are evaluated when the scale $k$ exits the Hubble radius. Anthropic constraints on $\zeta$ apply to scales $k \lesssim k_{\mathrm{eq}}$, where $k_{\mathrm{eq}}$ is the wave vector of the Hubble radius at matter-radiation equality. The potential $V$ evaluated when these scales first exit the Hubble radius may depend on $m_{\mathrm{P}}$ even when $V(\varphi)$ does not.

In our universe, $\zeta$ does not change appreciably with $k$, and we assume this holds at least approximately in other universes. Therefore we take $\zeta \approx \zeta\left(k_{\text {eq }}\right) \equiv \zeta_{\text {eq }}$ over all scales of interest. The scale $k_{\text {eq }}$ of matter-radiation equality itself depends on $m_{\mathrm{P}}$. However this dependence is logarithmic and its effect on $\zeta_{\text {eq }}$ is suppressed by the smallness of the slow-roll parameter, so we ignore it. Still, to solve for $\zeta_{\text {eq }}$ requires to choose a specific model for inflation. Since there is no standard model of inflation, we must be content with only a plausible range for the dependence on $m_{\mathrm{P}}$. We deduce this range by studying several of the most popular models of inflation. The results for chaotic inflation [31] with $V(\varphi) \propto \varphi^{p}$, for hybrid inflation [32], for natural inflation [33], and for ghost inflation [34] are listed in Table I.

The curvature perturbation $\zeta_{\text {eq }}$ may also be generated at the very end of inflation [35] or even much later as in the curvaton [36] and inhomogeneous reheating [37] scenarios. In each of these models a subdominant scalar $\chi$ receives fluctuations while the fluctuations to the inflaton are presumed to be negligible. The fluctuations in $\chi$ are then transferred to radiation either at the end of inflation,
TABLE I. The dependence of $\zeta_{\mathrm{eq}}$ on $m_{\mathrm{P}}$ for a variety of mechanisms to generate the curvature perturbation. The result for inhomogeneous reheating depends on the relative size between $\chi$ and other mass scales in the Lagrangian, and can interpolate between the two dependences given above.

\begin{tabular}{lc}
\hline \hline Mechanism to generate $\zeta_{\mathrm{eq}}$ & $m_{\mathrm{P}}$ dependence of $\zeta_{\mathrm{eq}}$ \\
\hline Inflation with $V(\varphi) \propto \varphi^{p}$ & $m_{\mathrm{P}}^{p / 2-2}$ \\
Natural inflation & $m_{\mathrm{P}}^{-3}$ \\
Hybrid inflation & $m_{\mathrm{P}}^{-3}$ \\
Ghost inflation & $m_{\mathrm{P}}^{-5 / 2}$ \\
End of inflation scenario & $\chi m_{\mathrm{P}}^{-3}$ \\
Curvaton scenario & $\chi^{-1} m_{\mathrm{P}}^{-1}$ \\
Inhomogeneous reheating & $\chi^{-1} m_{\mathrm{P}}^{-1}$ or $\chi m_{\mathrm{P}}^{-1}$ \\
\hline
\end{tabular}

during reheating, or during a phase transition much later. In each of these cases, the amplitude of the curvature perturbation depends on the local vacuum expectation value (vev) of $\chi$. The dependence of $\zeta_{\text {eq }}$ on $\chi$ and $m_{\mathrm{P}}$ when each of these mechanisms operates efficiently (that is, when any reheating occurs far out of equilibrium) is listed in Table I.

We note that these models are very flexible to anthropic selection. Although in principle $\chi$ may be set by interactions such that it is fixed among the set of universes we consider, in most cases $\chi$ is a stochastic variable over these universes. When $\zeta_{\mathrm{eq}}$ is generated at the end of inflation or via the curvaton or inhomogeneous reheating scenarios, then this implies that $\zeta_{\text {eq }}$ is also a stochastic variable over different universes. Therefore in these cases there exist universes with far different $m_{\mathrm{P}}$ but the same $\zeta_{\text {eq }}$ as in our universe, as well as universes with the same $m_{\mathrm{P}}$ but different $\zeta_{\text {eq }}$.

For future convenience we write $\zeta_{\text {eq }}$ in the form,

$$
\hat{\zeta}_{\mathrm{eq}} \approx \hat{m}_{\mathrm{P}}^{-\alpha},
$$

where according to our notation $\hat{\zeta}_{\text {eq }}$ is the curvature perturbation relative to its value in our universe. The various models of inflation that we studied suggest that we should restrict $\alpha$ to the range $0 \leq \alpha \leq 3$. However, the mechanisms that generate $\zeta_{\text {eq }}$ at the end of or well after inflation may generate a wide range of $\zeta_{\mathrm{eq}}$ for a wide range of $m_{\mathrm{P}}$. Although it is technically possible that keeping inflationary parameters fixed but varying $m_{\mathrm{P}}$ will cause the dominant contribution to the curvature perturbation to shift from one mechanism to another, this scenario should still be well approximated by the above guidelines so long as the variation in $m_{\mathrm{P}}$ is not too large.

\section{Reheating}

The reheating of the universe after inflation is achieved by coupling the inflaton to other degrees of freedom. In a typical model, after inflation the inflaton rocks within its potential well and redshifts like matter. The radiative decay 
products of the inflaton then dominate the energy density of the universe only after the Hubble rate falls below the decay width $\Gamma_{I}$. At this time the energy density in the inflaton is $\rho_{I}=3 m_{\mathrm{P}}^{2} \Gamma_{I}^{2}$. If $\Gamma_{I}$ is independent of $m_{\mathrm{P}}$, then the reheat temperature scales like

$$
\hat{T}_{\mathrm{RH}}=\hat{m}_{\mathrm{P}}^{1 / 2} .
$$

We have no empirical knowledge about reheating other than that $T_{\mathrm{RH}}$ is above the temperature of big bang nucleosynthesis. However, if our universe is described by a grand unified theory, then $T_{\mathrm{RH}}$ must be below the temperature of monopole production. In addition, if net baryon number is not generated during reheating, then $T_{\mathrm{RH}}$ must be high enough to support the dominant mechanism of baryogenesis. We comment on constraints like these in Sec. III.

\section{B. Baryogenesis}

We parametrize the net baryon number of a universe with the ratio between the number density of baryons and the number density of photons: $\eta \equiv n_{b} / n_{\gamma}$. Although we see no direct anthropic constraints on the value of $\eta$, it will enter into the anthropic constraints described in Secs. II E and II G. Presently, we seek to parametrize the dependence of $\eta$ on $m_{\mathrm{P}}$ for future reference. As with inflation, there is no standard model of baryogenesis. Therefore we must again content ourselves with only a range for the dependence on $m_{\mathrm{P}}$, based on the most plausible mechanisms. For a summary of these see, for example, the reviews of Refs. [38].

Perhaps the most plausible mechanism to produce net baryon number is leptogenesis [39]. For example, net lepton number is rather easily obtained by the out-ofequilibrium decay of a right-handed neutrino (RHN). The resulting lepton asymmetry can then be converted into net baryon number by sphaleron transitions within the standard model [40]. The value of $\eta$ that results from leptogenesis depends on how far out of equilibrium the RHN decays. If RHN decay occurs far out of equilibrium then the resulting baryon asymmetry $\eta$ is independent of $m_{\mathrm{P}}$. Otherwise, it scales roughly according to $\eta \propto m_{\mathrm{P}}^{-1}$. Note that baryogenesis via leptogenesis requires that the RHN, for example, be produced in the first place. We comment on this requirement in Sec. III.

The standard model of particle physics itself generates appropriate conditions for baryogenesis, when it is augmented by relatively light supersymmetric (SUSY) scalars to strengthen the electroweak phase transition. The process of electroweak baryogenesis is complex; yet interestingly it operates independently the scale of gravity. That is, although universal expansion is necessary to decrease the temperature of the universe and thus spur the electroweak phase transition, this process is relatively independent of the rate of temperature change. Therefore electroweak baryogenesis gives a baryon asymmetry that is independent of $m_{\mathrm{P}}$ [38].
Finally, we look at Affleck-Dine (AD) baryogenesis [41]. This mechanism takes advantage of scalar fields that possess baryon or lepton number, as would exist in SUSY or a grand unified theory (GUT). During inflation these fields may acquire large vevs, and then the influence of baryon nonconserving interactions on their subsequent evolution may generate significant baryon number. There are many models to implement AD baryogenesis and each may give a different dependence on $m_{\mathrm{P}}$. We simply give the result for a set of scenarios described in Ref. [42], where the scalar fields overlap a SUSY flat direction that is lifted by nonrenormalizable interactions and a negative induced mass term during inflation. To fit the resulting baryon asymmetry to observation requires different parametrizations when different nonrenormalizable interactions dominate; however in each case the dependence on $m_{\mathrm{P}}$ is given by $\eta \propto m_{\mathrm{P}}^{-3 / 2}$ [42].

For future reference, it is convenient to write the baryon to photon ratio in the form

$$
\hat{\eta} \approx \hat{m}_{\mathrm{P}}^{-\beta} .
$$

From the above discussion, we expect $0 \leq \beta \leq 3 / 2$, with perhaps the most plausible values being $\beta=0$ or $3 / 2$. As was the case with inflation, if more than one mechanism contributes to the net baryon number then we still expect the $\eta$ that results to be well approximated by the above guidelines.

\section{Big bang nucleosynthesis}

The process of big bang nucleosynthesis (BBN) populates the universe with light elements. In particular, the mass fractions of hydrogen $(X)$ and helium $(Y)$ are important for anthropic considerations described in Secs. II E and II F. Since we are only interested in $X$ and $Y$, we may take a very simplified view of BBN. Specifically, we assume that BBN generates appreciable concentrations of only hydrogen and helium-4. This is clearly appropriate within our universe, where the other products of BBN account for only about $0.01 \%$ of the mass fraction of the universe. Although this fraction may change significantly for differing values of $m_{\mathrm{P}}$, it would take a very large variation in $m_{\mathrm{P}}$ for this change to become significant next to $X$ or $Y$. A basic description of BBN can be found in Ref. [43].

When we approximate BBN to result in only hydrogen and helium- 4 , we require only the ratio of neutrons $(n)$ to protons $(p)$ to deduce $X$ and $Y$. Specifically,

$$
X \approx \frac{1-n / p}{1+n / p}, \quad Y \approx 1-X .
$$

A free neutron has $\Delta E \approx 1.3 \mathrm{MeV}$ more energy than a free proton. Thus if neutrons and protons are kept in thermal equilibrium by interactions that convert each into the other, then $n / p$ is given by the Boltzmann factor,

$$
n / p=\exp (-\Delta E / T)
$$


where $T$ is the temperature. Neutrons and protons are converted into each other via interactions such as $n+\nu \leftrightarrow$ $p+e$, where the $\nu$ denotes an electron neutrino and the $e$ an electron. In fact, the rates of these interactions decrease faster with temperature than does the Hubble rate. Therefore, below some temperature $T_{F}$ the universe expands too rapidly for, for example, an $n$ and a $\nu$ to find each other and convert into a $p$ and an $e$. Below this temperature the relative concentrations of $n$ and $p$ are fixed and the interaction is said to freeze out. ${ }^{1}$

The freeze-out temperature $T_{F}$ is obtained by equating the total rate of interactions converting neutrons to protons, $\Gamma_{\mathrm{np}}$, with the Hubble rate $H$. Then the neutron to proton ratio is $n / p \approx \exp \left(-\Delta E / T_{F}\right)$. Our universe contains $n / p \approx 1 / 7$ such that $X \approx 3 / 4$. Note that varying $m_{\mathrm{P}}$ so as to decrease $T_{F}$ works to decrease $n / p$ and therefore push $X$ closer to unity. Since in our universe $X \approx 3 / 4$, this effect is negligible at our level of analysis. On the other hand, for temperatures $T \geq T_{F}$ the rate of conversion between neutrons and protons is $\Gamma_{\mathrm{np}} \propto T^{5}$ and is independent of $\eta$. Since $H \propto T^{2} / m_{\mathrm{P}}$, the freeze-out temperature scales like $T_{F} \propto m_{\mathrm{P}}^{-1 / 3}$ when $T_{F}$ is larger than in our universe. Therefore we find,

$$
n / p \approx \exp \left[-\ln (7) \hat{m}_{\mathrm{P}}^{1 / 3}\right]
$$

The fractions $X$ and $Y$ are given by Eqs. (12). For example, when $\hat{m}_{\mathrm{P}}=10,5,0.2$, and 0.1 we have $X \approx 1,0.9,0.5$, and 0.4 , respectively.

\section{Matter domination}

We have assumed that the early universe is dominated by relativistic degrees of freedom, at least since BBN. However as the universe cools, massive degrees of freedom eventually become nonrelativistic. As it becomes nonrelativistic, the number density of this matter becomes exponentially suppressed relative to that of radiation. However, this dilution eventually causes matter to freeze out of equilibrium with the remaining radiation. Subsequently, the energy density of a massive species $i$ will redshift as $\rho_{i} \propto m_{i} n_{i}$ while the energy density in radiation scales as $\rho_{\text {rad }} \propto T n_{\text {rad. }}$. Thus it is inevitable that matter should ultimately come to dominate the energy density of the universe (structure formation constraints ensure that the cosmological constant does not become significant before matter domination).

\footnotetext{
${ }^{1}$ The decay of neutrons decreases $n / p$ from its value at $T_{F}$ by about $14 \%$ within our universe. This loss is determined by the time at which neutrons are efficiently captured into helium, which depends on $m_{\mathrm{P}}$ only via a logarithmic dependence on $\eta\left(m_{\mathrm{P}}\right)$ [43]. Inspecting Fig. 4.4 of Ref. [43], it can be shown that in the most extreme case of $\beta=3 / 2$, less than half of the neutrons decay for $\hat{m}_{\mathrm{P}} \lesssim 6$. On the other hand, for $\hat{m}_{\mathrm{P}} \gtrsim 6$ the helium fraction is less than a tenth of the hydrogen fraction. Therefore this effect is always negligible at our level of analysis.
}

Nevertheless, the energy density at matter-radiation equality and the fraction of matter in baryons are relevant to anthropic constraints described in Secs. II E and II G. In addition, our assumption that the early universe is radiation dominated does not hold if $\zeta$ is too large. In this case, primordial black holes may dominate the energy density of the universe while baryons are still relativistic. Then all of the baryons would be redshifted away or swallowed into black holes. This possibility is studied at the end of this section. In the following we neglect the neutrino content of the universe. Their influence on cosmology is commonly viewed as insignificant and we do not expect this to change since as a hot relic their density relative baryons is fixed. In addition, we assume the dark matter to be a WIMP. This allows for relatively precise predictions, as opposed to, for example, axion dark matter where the density is set by a stochastic variable [44]. (Note however that the stochastic nature of axion dark matter makes this possibility more flexible to anthropic selection, see, for example, Refs. [14,15].)

The energy density in a WIMP dark matter candidate is set by the relic abundance that results from the freeze-out of annihilation interactions when the temperature drops below the mass of the WIMP. After matter-radiation equality this gives the scaling,

$$
\rho_{\mathrm{cdm}} \propto m_{\mathrm{P}}^{-1} T^{3} .
$$

Meanwhile, after baryogenesis the relative abundance of baryons $\eta$ is conserved. Therefore at temperatures below the nucleon mass the energy density in baryons scales like

$$
\rho_{b} \propto \eta T^{3} \text {. }
$$

Using that in our universe $\rho_{b} / \rho_{\text {cdm }} \approx 1 / 5$ and that the energy density in radiation scales as $\rho_{\text {rad }} \propto T^{4}$, we find the energy density at matter-radiation equality to be

$$
\hat{\rho}_{\mathrm{eq}} \approx\left(\frac{1}{6} \hat{\eta}+\frac{5}{6} \hat{m}_{\mathrm{P}}^{-1}\right)^{4} .
$$

Finally, we note the baryon fraction within matter,

$$
\hat{f}_{b} \approx\left(\frac{1}{6}+\frac{5}{6} \hat{\eta}^{-1} \hat{m}_{\mathrm{P}}^{-1}\right)^{-1} .
$$

As described above, these results do not hold if $\zeta$ is so large as to produce an abundance of primordial black holes (PBHs) [45]. Numerical analysis reveals that a PBH is formed when an energy density fluctuation $\sigma \gtrsim 0.7$ enters the Hubble radius [46]. Meanwhile, during radiation domination $\sigma=\frac{4}{3} \zeta$ at Hubble radius crossing [47]. Therefore we require $\zeta(k) \lesssim 0.5$ in order to prevent the formation of a PBH when the scale $k$ enters the Hubble radius. In fact, this implies a somewhat stronger constraint on $\zeta$. This is because $\zeta$ is a stochastic variable with a Gaussian tail and because a PBH need not be formed each Hubble time in order for PBHs to dominate the energy density of the universe. This constraint is worked out in Ref. [19] and we follow that analysis. 
The likelihood that a curvature perturbation with root mean square $\zeta$ is greater than or equal to $1 / 2$ is $f(\zeta)=$ $\operatorname{erfc}\left(2^{-3 / 2} \zeta^{-1}\right)$, where the complementary error function is defined $\operatorname{erfc}(x) \equiv 2 \pi^{-1 / 2} \int_{x}^{\infty} e^{-z^{2}} d z$. PBHs redshift like matter while the other degrees of freedom redshift like radiation. Therefore by matter-radiation equality $\mathrm{PBHs}$ will compose roughly $\left(a_{\mathrm{eq}} / a_{\mathrm{pbh}}\right) f$ of the energy density of the universe. Here $a_{\mathrm{pbh}}$ is the scale factor at which newly formed PBHs have sufficient mass to persist until equality. Then PBHs do not dominate the energy density of the universe when

$$
\operatorname{erfc}\left(2^{-3 / 2} \zeta_{\mathrm{eq}}^{-1}\right) \lesssim \frac{a_{\mathrm{pbh}}}{a_{\mathrm{eq}}},
$$

where we have neglected any tilt in $\zeta$. This approximation underestimates the largest $\zeta_{\text {eq }}$ since recent observations suggest a negative tilt [48].

The erfc function depends very strongly on its argument; therefore the $m_{\mathrm{P}}$ dependence of the ratio $a_{\mathrm{pbh}} / a_{\mathrm{eq}}$ is inconsequential for our analysis. As one looks to earlier times, the lifetimes of PBHs decrease more rapidly than cosmic time decreases. Therefore Eq. (19) ensures that PBHs dominate at no time prior to equality. Solving for when PBH lifetimes equal about 70000 years gives $a_{\mathrm{pbh}} / a_{\mathrm{eq}} \sim$ $10^{-20}$ which gives $\zeta_{\text {eq }} \lesssim 6 \times 10^{-2}$. Translating this into a constraint on $m_{\mathrm{P}}$, we find

$$
\hat{m}_{\mathrm{P}}^{\alpha} \gtrsim 7 \times 10^{-4},
$$

where we have used that the density fluctuation at Hubble radius crossing is $\sigma \approx 5 \times 10^{-5}$ [48]. Equation (20) is always weaker than the stellar lifetime constraint of Sec. II F.

\section{E. Structure formation}

The formation of structure within our universe occurs in several stages. First, overdensities in the nearly pressureless dark matter begin to grow upon entry into the Hubble radius. During radiation domination, this growth is logarithmic with time, while after the dark matter comes to dominate the energy density of the universe overdensities grow in proportion to the growth in the cosmic scale factor. On the other hand, overdensities in the baryons cannot grow until after recombination. However, within an efold or so after recombination they have grown to match the overdensity in dark matter, and subsequently grow in proportion to the growth in the cosmic scale factor. When these overdensities have grown sufficiently they separate from the Hubble flow and virialize to form what are termed halos.

After virialization, the cold dark matter within halos is stabilized against gravitational collapse by its inability to release its kinetic energy. However, the baryons within the halo must collapse beyond their initial virialization radius if they are to fragment and condense into galaxies and ultimately into stars. This requires that the baryons have a means to dissipate their thermal energy. The constraints on $m_{\mathrm{P}}$ that are implied by these stages of structure formation are discussed in the sections below. Presently, we describe the initial growth in over-densities for future reference. The subject of galaxy formation and, in particular, star formation is complex and not yet fully understood. We rely heavily on the simplifying assumptions and models of Refs. [15,19].

We find it convenient to track the evolution of overdensities in position space, as opposed to Fourier space. At matter-radiation equality, the variance of energy density fluctuations over scales with comoving radius $R$ is

$$
\sigma_{\text {eq }}^{2}(R)=\int_{0}^{\infty} \frac{d k}{2 \pi^{2}} k^{2} W(k R) T(k) P(k),
$$

where $W$ is a window function that may be chosen to be a "top hat" with radius $R, T$ is a transfer function to account for the evolution of perturbations between when they enter the Hubble radius and equality, and $P$ is the primordial power spectrum of fluctuations, $P(k) \propto\left\langle\zeta(k)^{2}\right\rangle$. We parametrize a comoving scale with radius $R$ according to the total mass $\mu$ that is enclosed within a sphere of radius $R$. In addition, we measure $\mu$ relative to the mass of our galaxy (more precisely the mass of our galaxy plus its dark matter halo); thus $\mu=1$ corresponds to $10^{12} M_{\odot}$, where $M_{\odot}$ is the mass of the sun.

A numerical curve fit to Eq. (21) gives [15],

$$
\sigma_{\mathrm{eq}} \simeq 1.45 \times 10^{-3} s(\mu) \hat{\zeta}_{\mathrm{eq}}\left(m_{\mathrm{P}}\right),
$$

where the function $s(\mu)$ carries the scale dependence of $\sigma_{\text {eq }}$. This scale dependence occurs because at the time of equality smaller scales have been within the Hubble radius for a longer time than larger scales. The function $s(\mu)$ is equivalent to Eq. (A13) in Ref. [15]. However, we have normalized $s(\mu)$ such that $s(1)=1$. In addition, we define the variable $\mu$ with respect to a different scale than the authors of Ref. [15]. Therefore within this paper $s$ is given by

$$
\begin{aligned}
s(\mu)= & {\left[\left(0.76 \ln \left[17+\mu^{-1 / 3}\right]-0.22\right)^{-0.27}\right.} \\
& \left.+0.17 \mu^{0.18}\right]^{-3.7} .
\end{aligned}
$$

Note that $s$ is a decreasing function of $\mu$. In addition, we emphasize that $\sigma_{\text {eq }}$ is the root mean square (rms) value of a Gaussian random field. Therefore constraints involving $\sigma_{\text {eq }}\left(\right.$ or $\hat{\zeta}_{\text {eq }}$ ) are never sharp in the sense that they may be overcome by fluctuations that happen to be larger or smaller than is typical.

After recombination but before the domination of cosmological constant, a linear overdensity is given by [9]

$$
\sigma \approx\left(\frac{2}{5}+\frac{3}{5} \frac{a}{a_{\mathrm{eq}}}\right) \sigma_{\mathrm{eq}} .
$$

Soon after recombination the first term is negligible. 
Equation (24) is accurate until cosmological constant domination, after which $\sigma$ grows by another factor of about 1.44 and then stops. An overdensity separates from the Hubble flow and virializes when a linear analysis gives $\sigma=1.69$ [49]. Thus the cosmic mean energy density at virialization is

$$
\rho_{*} \approx\left(\frac{3}{5} \frac{\sigma_{\mathrm{eq}}}{1.69}\right)^{3} \rho_{\mathrm{eq}} \approx 1.4 \times 10^{-10} \rho_{\mathrm{eq}} \hat{\zeta}_{\mathrm{eq}}^{3} s^{3} .
$$

The energy density within the condensed halo is larger by roughly a factor of $18 \pi^{2}$. We denote this as

$$
\rho_{\text {vir }} \approx 18 \pi^{2} \rho_{*} \approx 2.4 \times 10^{-8} \rho_{\text {eq }} \hat{\zeta}_{\text {eq }}^{3} s^{3} .
$$

Note that these quantities depend on both $m_{\mathrm{P}}$ and the mass scale $\mu$ of the virialized halo.

The above description of halo formation relies on three important aspects of the standard cosmology: we assume that the dark matter density dominates over the baryon density, that recombination occurs before the virialization of the dark matter halo, and that virialization occurs before the domination of cosmological constant. Enforcing the above conditions implies constraints on $m_{\mathrm{P}}$. These are discussed in the next section. We discuss the possibility for a nonstandard path toward structure formation in Appendix A.

\section{Halo virialization}

Before proceeding to galaxy formation, we must ensure that overdensities separate from the cosmic expansion and virialize before the domination of the cosmological constant halts their growth. As mentioned above, an overdensity $\sigma$ has separated from the Hubble flow when a linear analysis gives $\sigma \geq 1.69$. On the other hand, the maximum size that is reached by a rms linear overdensity is given by

$$
\sigma_{\infty} \approx 1.44 \times \frac{3}{5} \frac{a_{\Lambda}}{a_{\mathrm{eq}}} \sigma_{\mathrm{eq}} \approx 3.20 \hat{\rho}_{\mathrm{eq}}^{1 / 3} \hat{\zeta}_{\mathrm{eq}} s,
$$

where $a_{\Lambda}$ is the scale factor at $\rho_{\Lambda}$ domination. Therefore the requirement that $\sigma_{\infty} \geq 1.69$ gives

$$
\hat{\rho}_{\text {eq }} \hat{\zeta}_{\text {eq }}^{3} s^{3} \gtrsim 0.1 \text {. }
$$

Since with a larger value for $m_{\mathrm{P}}$ structures form later, this is a constraint against increasing $m_{\mathrm{P}}$. Substituting previous results into Eq. (28) gives

$$
\left(\frac{1}{6} \hat{m}_{\mathrm{P}}^{-\beta}+{ }_{6}^{5} \hat{m}_{\mathrm{P}}^{-1}\right)^{4} \hat{m}_{\mathrm{P}}^{-3 \alpha} s(\mu)^{3} \gtrsim 0.1 .
$$

The curves that saturate this inequality for various choices of $\alpha$ and $\beta$ are displayed in Fig. 1 under the label " $\sigma_{\infty}=1.69$." In light of these plots, our value of $m_{\mathrm{P}}$ may be construed as nearly saturating this constraint. However, this perception derives from the strong $m_{\mathrm{P}}$ dependence of $\rho_{\text {vir }}$, and not from our galaxy being at the edge of saturating the Weinberg bound. In addition this constraint, analogous to many others below, holds for a rms fluctuation $\sigma_{\mathrm{eq}}$ but is weaker for larger fluctuations. For these reasons we emphasize that although Eq. (29) does not allow for $m_{\mathrm{P}}$ to be increased significantly, it is still true that our value of $m_{\mathrm{P}}$ is not at the edge of the anthropic range. Since the existence of an anthropically allowed window surrounding our value of $m_{\mathrm{P}}$ is essential to the arguments of Sec. III, we provide a more elaborate discussion of this boundary in Appendix B. This may serve as an illustration of how "soft" are other constraints that depend on $\sigma_{\text {eq }}$.

The above analysis assumes that halos virialize at least an e-fold or so after recombination. This is to ensure that baryons may collapse into the dark matter potential wells and participate in the virialization. Thus we require $\rho_{*} / \rho_{\text {rec }} \lesssim e^{-3}$. The energy density at recombination is set by the temperature of recombination, which depends only logarithmically on $m_{\mathrm{P}}$ and $\eta$. We ignore this logarithmic dependence and take $T_{\text {rec }} \approx 3000 \mathrm{~K}$ in every universe that we consider. Using that at any time after equality $\rho_{*} \propto$ $\rho_{\text {rec }} \propto T^{3}$, we find for this constraint,

$$
\hat{\rho}_{\text {eq }}^{-3 / 4} \hat{\zeta}_{\text {eq }}^{-3} s^{-3} \gtrsim 8 \times 10^{-8} .
$$

Since with a smaller value of $m_{\mathrm{P}}$ the matter energy density at equality and the amplitude of density perturbations are both larger, this is a constraint against decreasing $m_{\mathrm{P}}$. In terms of $\hat{m}_{\mathrm{P}}$ and $\mu$, this gives

$$
\left(\frac{1}{6} \hat{m}_{\mathrm{P}}^{-\beta}+\frac{5}{6} \hat{m}_{\mathrm{P}}^{-1}\right)^{-3} \hat{m}_{\mathrm{P}}^{3 \alpha} s(\mu)^{-3} \gtrsim 8 \times 10^{-8} .
$$

The curves that saturate this constraint for various $\alpha$ and $\beta$ are displayed in Fig. 1 with the label " $t_{\mathrm{rec}}=e^{-2 / 3} t_{\mathrm{vir}}$ "

Finally, we require that dark matter dominate over baryonic matter so that dark matter potential wells are deep enough to condense baryon overdensities after recombination. This simply translates into the constraint $f_{b} \lesssim 1 / 2$, which gives

$$
\begin{array}{ll}
\hat{m}_{\mathrm{P}} \lesssim 5^{1 /(1-\beta)} & \text { for } \beta<1, \\
\hat{m}_{\mathrm{P}} \gtrsim 5^{1 /(1-\beta)} & \text { for } \beta>1 .
\end{array}
$$

For $\beta=1$ this argument provides no constraint on $m_{\mathrm{P}}$ since in that case $f_{b}$ is independent $m_{\mathrm{P}}$.

\section{Galaxy formation}

Although the dark matter within a halo cannot dissipate its kinetic energy to further collapse, the baryons may do so via electromagnetic interactions. If the cooling time scale $\tau_{\text {cool }}$ is less than the time scale of gravitational dynamics $\tau_{\text {grav }}$, then not only do the baryons collapse, but perturbations in the baryon density fragment into smaller structures. These structures ultimately fall into a rotationally supported disk. Perturbations may further fragment if the disk satisfies the Jeans instability criteria, which is that $\tau_{\text {grav }}$ be less than the time it takes for a pressure wave to traverse the perturbation. Fragmentation continues until perturbations become Jeans-stable and overdensities relax 
adiabatically into hot balls of gas. This appears to be the path by which halos within our universe ultimately condense into galaxies of stars (for background see, for example, Ref. [50]).

In order to ensure galactic dynamics similar to those within our universe, one might therefore first impose that for typical halos, $\tau_{\text {cool }} \leqslant \tau_{\text {grav }}{ }^{2}$ We take the dynamical time scale of the halo to be the time it would take for a test particle to free-fall to the center of the halo. For a spherical halo of constant density, this is

$$
\tau_{\mathrm{grav}} \approx \sqrt{\frac{3}{2} \pi^{2}} m_{\mathrm{P}} \rho_{\mathrm{vir}}^{-1 / 2}
$$

For the cooling time scale we use the total thermal energy divided by the rate of energy loss, per unit volume:

$$
\tau_{\text {cool }} \approx \frac{3}{2} \frac{f_{b} \rho_{\mathrm{vir}}}{m_{N} \mu_{b}} \frac{T_{\mathrm{vir}}}{\Lambda_{c}},
$$

where $\mu_{b}$ is the mean molecular weight of the baryons in the halo (in units of the nucleon mass $m_{N}$ ), $T_{\text {vir }}$ is the mean temperature of the halo, and $\Lambda_{c}$ is the rate of energy loss per unit volume. The quantity $f_{b} \rho_{\text {vir }} / m_{N} \mu_{b}$ is the baryon number density, including electrons.

The mean molecular weight depends on the ionization fraction and the hydrogen mass fraction of the halo. For example, for a fully ionized halo we have

$$
\mu_{b} \approx \frac{n_{\mathrm{H}}+4 n_{\mathrm{He}}}{n_{e}+n_{\mathrm{H}}+n_{\mathrm{He}}} \approx \frac{4}{3+5 X},
$$

where the subscripts denote electrons, hydrogen, or helium. Note that $\mu_{b}$ never strays more than a factor of 2 from unity. To estimate the temperature of the halo, we first note that in a virialized halo the mean kinetic energy equals half the mean gravitational binding energy. Thus for a halo of mass $M$ we write $M v_{\text {vir }}^{2} \approx \frac{3}{40 \pi} m_{\mathrm{P}}^{-2} M^{2} R^{-1}$, where $v_{\text {vir }}$ is a characteristic velocity for virialized particles and $R$ is the radius of the halo. Since $M \approx \frac{4 \pi}{3} R^{3} \rho_{\text {vir }}$ and since $T_{\text {vir }} \approx$ $\frac{1}{3} \mu_{b} m_{N} v_{\text {vir }}^{2}$, we obtain

\footnotetext{
${ }^{2}$ We note that more careful considerations involving galactic dynamics may suggest a far weaker constraint than the one we pursue. We require $\tau_{\text {cool }} \lesssim \tau_{\text {grav }}$, where each time scale is evaluated at virialization. However, the baryons within a halo will cool even if this condition is not satisfied. As described in Ref. [51], this cooling pushes the gas of baryons along a curve in the temperature-density phase space that eventually leads to the condition $\tau_{\text {cool }} \lesssim \tau_{\text {grav }}$ being satisfied, albeit at a much later time. It is then necessary to consider any other factors that might constraint the time scale $\tau_{\text {cool }}$. Reference [15] has pointed out that if $\tau_{\text {cool }}$ is not much smaller than the Hubble time, then baryons do not cool significantly before being reheated by halo mergers. Eventually halo merging ceases due to cosmological constant domination. However, even then, one must worry about too large a faction of baryons evaporating from the halo before they cool sufficiently to sink deeper into the gravitational potential well [15]. These considerations are beyond the scope of this paper.
}

$$
T_{\mathrm{vir}} \approx \frac{1}{78} \mu_{b} m_{N} m_{\mathrm{P}}^{-2} M^{2 / 3} \rho_{\mathrm{vir}}^{1 / 3} .
$$

Note that $T_{\text {vir }} \propto \sigma_{\text {eq }}$ so that $T_{\text {vir }}$ is a stochastic variable for halos of a given mass.

The baryons within a halo may cool via Compton scattering, bremsstrahlung, the excitation of hydrogen or helium lines, in addition to other mechanisms. These all contribute to the rate of thermal energy dissipation $\Lambda_{c}$. Thus $\Lambda_{c}$ is a complicated function of temperature, which also depends on the halo composition and therefore the hydrogen and helium fractions $X$ and $Y$. Rather than attempt an estimate of $\Lambda_{c}$, we use the cooling rates given in Refs. [52]. These include the processes listed above, but we neglect the possibility for molecular cooling, which is insignificant at the temperatures we consider. The galactic cooling constraint $\tau_{\text {cool }} \lesssim \tau_{\text {grav }}$ is now

$$
m_{\mathrm{P}}^{3} \Lambda_{c} f_{b}^{-1} M^{-2 / 3} \rho_{\mathrm{vir}}^{-11 / 6} \gtrsim 5 \times 10^{-3} .
$$

The curves that saturate this inequality are displayed in Fig. 1 with the label " $\tau_{\text {cool }}=\tau_{\text {grav }}$."

As noted in Ref. [15], if a galaxy contains too little mass then early supernovae may blow away a significant fraction of its baryons when they explode. We expect the effects of a supernova to be relatively localized if the gravitational binding energy of the galaxy by far exceeds the energy released in the supernova [15]. This can be ensured by requiring that the energy released in the supernova be less than the halo binding energy,

$$
E_{\text {bind }} \approx \frac{3}{40 \pi m_{\mathrm{P}}^{2}} \frac{M^{2}}{R} \approx \frac{1}{26} m_{\mathrm{P}}^{-2} M^{5 / 3} \rho_{\mathrm{vir}}^{1 / 3} .
$$

Note that the baryons within a galaxy are much more tightly bound than when in the original halo (see, for example, the galactic disk estimates of Secs. IIE 3 and II G). Therefore Eq. (38) is a significant underestimate of the binding energy of a galaxy.

We expect the energy released in a supernova to scale roughly as the binding energy of a Chandrasekhar mass at its Schwarzschild radius [15], or as the binding energy of a typical star, both of which are proportional to $m_{\mathrm{P}}^{3}$ (see Sec. II F). Thus we write this energy $E_{\mathrm{sn}} \hat{m}_{\mathrm{P}}^{3}$, where $E_{\mathrm{sn}} \approx$ $10^{51}$ erg is the typical supernova energy within our universe. Requiring that $E_{\text {bind }} \gtrsim E_{\text {sn }}$ gives

$$
\hat{m}_{\mathrm{P}}^{-5} \hat{\rho}_{\text {eq }}^{1 / 3} \hat{\zeta}_{\text {eq }} \mu^{5 / 3} s \gtrsim 4 \times 10^{-9} .
$$

Since halos of a given mass become more weakly gravitationally bound as $m_{\mathrm{P}}$ is increased, Eq. (39) is a constraint against increasing $m_{\mathrm{P}}$. After inserting previous results this becomes

$$
\left(\frac{1}{6} \hat{m}_{\mathrm{P}}^{-\beta}+\frac{5}{6} \hat{m}_{\mathrm{P}}^{-1}\right)^{4 / 3} \hat{m}_{\mathrm{P}}^{-5-\alpha} \mu^{5 / 3} s(\mu) \gtrsim 4 \times 10^{-9} .
$$

The curves that saturate Eq. (40) are displayed in Fig. 1 under the label " $E_{\mathrm{bind}}=E_{\mathrm{sn}}$." 


\section{Star formation}

If the above conditions are met, the baryons in a halo will radiate away energy and settle into a disk supported by its angular momentum. We then require that the disk fragment so that ultimately stars may form [15]. The stability of galactic disks against both radial and vertical perturbations can be studied using a standard Jeans analysis, which compares the dynamical time scale $\tau_{\text {grav }}$ to the time it takes a pressure wave to traverse the perturbation. It turns out that the stability criteria for the two modes differ by only an order unity coefficient [15,50,53]. In Ref. [53] it is shown that for perturbations in the vertical direction, perturbations are unstable when the total mass of the disk satisfies,

$$
M_{\text {disk }} \geq 120 m_{\mathrm{P}}^{2} v_{p} v_{c} R_{\text {disk }},
$$

where $v_{p}$ is the typical peculiar velocity of particles in the disk, $v_{c}$ is the circular velocity of these particles, and $R_{\text {disk }}$ is the disk radius.

The mass of the disk is simply the mass of the baryons in the halo, $M_{\text {disk }}=f_{b} M$. Meanwhile, the peculiar velocity is related to the temperature of the disk. This temperature will be the lowest temperature to which the baryons can cool as they collapse, which is roughly set by the hydrogen line temperature $T_{\mathrm{H}} \approx 10^{4} \mathrm{~K} .^{3}$ Therefore the typical peculiar velocity may be written

$$
v_{p} \approx \sqrt{\frac{3 T_{\mathrm{H}}}{\mu_{b} m_{N}}} .
$$

The circular velocity $v_{c}$ is deduced by conserving angular momentum as the baryonic halo collapses. On the one hand, the disk angular momentum can be roughly written as $R_{\text {disk }} M_{\text {disk }} v_{c}$. On the other hand, the baryons in a halo start with angular momentum $\frac{1}{\sqrt{8 \pi}} f_{b} \lambda m_{\mathrm{P}}^{-1} M^{3 / 2} R^{1 / 2}$, where $\lambda$ is the dimensionless spin parameter [54],

$$
\lambda \equiv \sqrt{8} \pi \frac{J E_{\mathrm{bind}}}{m_{\mathrm{P}} M^{5 / 2}},
$$

where $J$ is the magnitude of the angular momentum, $E_{\text {bind }}$ is the gravitational binding energy, and all of the above quantities are evaluated for the original halo. Before the gravitational collapse of the baryons out of the dark matter halo, it is reasonable to assume that the angular momentum of the baryons and dark matter are equally distributed according to mass, such that initially $J_{b}=M_{b} J_{h} / M_{h}$ where the subscripts $b$ and $h$ refer to baryonic and dark matter halo quantities [53]. Then assuming that angular momentum is conserved as the baryons in the halo col-

\footnotetext{
${ }^{3}$ Although the rate of galactic cooling is reduced below the temperature of hydrogen line freezeout, cooling still proceeds via molecular transitions in, for example, $H_{2}$. Therefore $T_{\min }$ may become very small if one is willing to wait a long time before disk fragmentation. We follow Ref. [15] and study when galactic dynamics are similar to those within our universe.
}

lapse, we find

$$
v_{c} \sim \frac{\lambda}{\sqrt{8 \pi}} m_{\mathrm{P}}^{-1} M^{1 / 2} R^{1 / 2} R_{\text {disk }}^{-1} .
$$

We do not require to solve for $R_{\text {disk }}$ after this expression for $v_{c}$ is inserted into Eq. (41).

Equation (41) can now be written in the simple form [15]

$$
f_{b} \gtrsim 4 \lambda\left(\frac{T_{\mathrm{H}}}{T_{\mathrm{vir}}}\right)^{1 / 2} \text {. }
$$

The spin parameter $\lambda$ is different for different halos, but it is roughly independent of $m_{\mathrm{P}}, \mu$, and the amplitude of density perturbations, and it typically lies near $\lambda \approx 0.05$ [54]. Substituting into Eq. (45) gives

$$
\hat{\mu}_{b}^{1 / 2} \hat{f}_{b} \hat{m}_{\mathrm{P}}^{-1} \hat{\rho}_{\mathrm{eq}}^{1 / 6} \hat{\zeta}_{\mathrm{eq}}^{1 / 2} \mu^{1 / 3} s^{1 / 2} \gtrsim 0.2 .
$$

This results in a constraint against increasing $m_{\mathrm{P}}$. We use previous results to convert this into a constraint on $\hat{m}_{\mathrm{P}}$ and $\mu$, which gives

$$
\left(\frac{1}{6} \hat{m}_{\mathrm{P}}^{-\beta}+\frac{5}{6} \hat{m}_{\mathrm{P}}^{-1}\right)^{-1 / 3} \hat{m}_{\mathrm{P}}^{-1-\beta-\alpha / 2} \mu^{1 / 3} s^{1 / 2} \geq 0.2,
$$

where $\hat{\mu}_{b}\left(\hat{m}_{\mathrm{P}}\right)$ depends relatively weakly on $m_{\mathrm{P}}$ and has been ignored. The curves that saturate this constraint for various $\alpha$ and $\beta$ are displayed in Fig. 1 with the label "disk inst."

It is essential that at some point fragmentation ceases so that overdensities can smoothly collapse into a star. The process of fragmentation may be seen to terminate when individual fragments become sufficiently opaque so as to trap most of their radiation [55]. In the Jeans picture, this allows the temperature of a perturbation to rise and correspondingly increase the sound speed and thus prevent further fragmentation. As described in Ref. [55], the mass scale at which this occurs is relatively independent of the dominant contributions to the cooling rate and opacity and gives a typical stellar mass that scales like $m_{\mathrm{P}}^{3}$. Interestingly, this is the same scaling behavior that restricts the sizes of stars based on their internal temperature being high enough to fuse hydrogen and their radiation pressure being low enough so as to not blow the star apart. We elaborate on this in the next section.

\section{F. Stellar dynamics}

We have so far ensured that the fragmentation of overdensities persists on all scales greater than a relatively small scale that is roughly proportional to $m_{\mathrm{P}}^{3}$. We now require that the temperature within some of the remaining structures is sufficient to fuse hydrogen to form a star. It is possible that the mere existence of stars is not a sufficient condition for the existence of observers. Therefore we also consider the requirement that some of these stars supernova in order to generate heavy elements. In addition, we consider the requirement that some of these stars have both a surface temperature within a factor of 2 that of the sun 
and a main-sequence lifetime of at least a few billion years. Our motivation for selecting these specific criteria is simple. Without knowing what are the necessary conditions for observers to arise within a stellar system, we study what seem at least to be two sufficient conditions.

Reference [16] has studied the basic requirements that constrain the properties of stars. For decreasing stellar mass, the central temperature must be above some minimum temperature $T_{\text {nuc }}$ that is necessary to fuse hydrogen. The central temperature within a low-mass star is estimated by balancing the influences of gravitational pressure and electron degeneracy. This gives for the central temperature of a low-mass star [16],

$$
T_{c} \propto m_{\mathrm{P}}^{-4} M^{4 / 3},
$$

where $M$ is the stellar mass. The least massive stars have $T_{c}=T_{\text {nuc }}$. Since $T_{\text {nuc }}$ is independent of $m_{\mathrm{P}}$, in any universe these stars have mass

$$
M_{\min } \propto m_{\mathrm{P}}^{3} .
$$

On the other hand, there is also an upper limit to the mass of a star. If the radiation pressure within a star well exceeds the gravitational pressure that its mass can provide, then the star itself becomes unstable upon the ignition of its core. This constrains the maximum mass that a star may have to satisfy the scaling [16],

$$
M_{\max } \propto m_{\mathrm{P}}^{3} .
$$

Note that the minimum and maximum mass of a star both scale as $m_{\mathrm{P}}^{3}$. This is also the scaling of the typical mass that becomes sufficiently opaque to prevent further fragmentation. This means that, given a window of masses for which stars exist in our universe, there will also be such a window within universes with significantly different values of $m_{\mathrm{P}}$.

\section{Stellar lifetimes and spectra}

We now seek constraints to ensure that some of the stars produced within a particular universe have surface temperatures and main-sequence lifetimes that appear to be sufficient for the evolution of observers. Our purpose in investigating this condition is to ensure that we do not overlook what might be viewed as an important anthropic constraint. Therefore we adopt a very restrictive perspective and require that some stars have surface temperatures of at least $3500 \mathrm{~K}$ and that these stars have main-sequence lifetimes greater than the time scale of biological evolution, $\tau_{\text {evol }} \approx 5 \times 10^{9} \mathrm{yrs}$. This surface temperature is chosen in part to simplify the calculation of stellar lifetimes and in part because a black body at this temperature radiates a significant fraction of its power into the frequency band accessible to chemistry. The evolutionary time scale $\tau_{\text {evol }}$ should be understood to include the time required for a planet to condense and cool, minus the time it takes the star to reach main-sequence hydrogen burning.
This time may be different for different planets, but we do not expect it to form the dominant contribution to $\tau_{\text {evol }}$.

The main-sequence lifetime of a star is roughly equal to the available energy of the star divided by the typical rate that it radiates energy away,

$$
\tau_{\star} \propto X M L^{-1} .
$$

Here $L$ is the typical luminosity of the star during main sequence and $X$ is the hydrogen fraction. We assume that differences in composition other than differences in the hydrogen fraction have benign consequences. In addition, we neglect the $m_{\mathrm{P}}$ dependence of the mean molecular weight $\mu_{\star}$, since $\mu_{\star}$ changes by only roughly a factor of 2 as $X$ ranges from zero to unity. For an introduction to the major concepts of stellar astrophysics, we have found useful Refs. [56-58].

Our narrow purpose allows for a simplified analysis of the necessary stellar dynamics. Since we specify stars by their surface temperature, we write the luminosity $L \propto$ $R^{2} T_{s}^{4}$ for stellar radius $R$ and surface temperature $T_{s}$. To eliminate $R$, we note that the central temperature of a star scales as

$$
T_{c} \propto \frac{1}{m_{\mathrm{P}}^{2}} \frac{M}{R} .
$$

Thus we can write

$$
\tau_{\star} \propto \frac{X m_{\mathrm{P}}^{4} T_{c}^{2}}{M T_{s}^{4}} .
$$

The lifetime of a star is maximized by considering the minimum allowed surface temperature, in this case $T_{s}=$ $3500 \mathrm{~K}$. Since both the lower end and the upper end of the window for stellar masses scale as $m_{\mathrm{P}}^{3}$, as a basic approximation we may take this to be the scaling for all stellar masses at fixed $T_{s}$ and $T_{c}$. Combining this with Eqs. (51)(53) gives

$$
\hat{\tau}_{\star} \approx \hat{X} \hat{m}_{\mathrm{P}}
$$

where $\hat{\tau}_{\star}$ is measured in units of the main-sequence lifetime of these stars within our universe. This lifetime is roughly $100 \times 10^{9}$ years [59]. Therefore the constraint $\tau_{\star} \gtrsim \tau_{\text {evol }}$ becomes

$$
\hat{X} \hat{m}_{\mathrm{P}} \gtrsim 5 \times 10^{-2} .
$$

This requires that $\hat{m}_{\mathrm{P}}$ satisfy $\hat{m}_{\mathrm{P}} \gtrsim 0.1$.

We now consider this analysis in a little more detail. In particular, we consider the effects of convection and electron degeneracy explicitly in order to motivate that we can keep $T_{s} / T_{c}$ fixed while scaling $m_{\mathrm{P}}$ and that the stellar mass scales like $m_{\mathrm{P}}^{3}$ at fixed $T_{c}$. An ionized star in which radiation pressure can be neglected and in which the energy transport is everywhere dominated by convection is well approximated as a polytrope with polytropic index $i=3 / 2$ $[56,58]$. This implies certain scalings between stellar prop- 
erties and, in particular, that for these stars $T_{s} / T_{c}$ is independent of $m_{\mathrm{P}}, M$, and $R$. Within our universe, stars with surface temperatures at and below $3500 \mathrm{~K}$ have masses $M \lesssim 0.35 M_{\odot}$ and are well approximated by these polytropes. In addition, it can be shown that stars defined by these temperatures remain convection dominated as $m_{\mathrm{P}}$ is decreased [58]. To see this intuitively, note that convection is driven by tidal forces and, all else being equal, one expects the tidal forces within a star to increase as $m_{\mathrm{P}}$ is decreased. Therefore, we expect $T_{s} / T_{c}$ to be fixed for stars with $T_{s}=3500 \mathrm{~K}$ as $m_{\mathrm{P}}$ is decreased.

Meanwhile, so long as electron degeneracy is significant within the center of the star, the mass required to achieve a fixed central temperature scales like $m_{\mathrm{P}}^{3}$ [16]. Stars with a surface temperature of $3500 \mathrm{~K}$ are indeed partially degenerate within our universe, but they could become nondegenerate after some amount of scaling $M \propto m_{\mathrm{P}}^{3}$. To see that there can exist stars for which the degeneracy remains fixed, consider again the polytrope model. The electron degeneracy at the center of a star is a function of the ratio $n_{e} T_{c}^{-3 / 2}$, where $n_{e}$ is the number density of electrons. The scaling relations applicable to an $i=3 / 2$ polytrope imply that the electron density $n_{e}$ scales like the average density of the star, which at constant central temperature scales like $m_{\mathrm{P}}^{6} M^{-2}$. Next note that the electron degeneracy at the center of a star is a constant if the mass of the star scales as $M \propto m_{\mathrm{P}}^{3}$ for fixed central temperature $T_{c}$. This is precisely the scaling that describes a partially degenerate star, which means that a partially degenerate star remains partially degenerate as $m_{\mathrm{P}}$ is scaled while keeping $T_{c}$ fixed. Therefore we expect stars with a surface temperature of $3500 \mathrm{~K}$ to remain partially degenerate as we decrease $m_{\mathrm{P}}$ keeping $T_{s}$ fixed, so that indeed $M \propto m_{\mathrm{P}}^{3}$. These arguments justify the constraint equation (55).

It is illuminating to consider a different form of analysis. This applies to ionized stars where radiation pressure can be neglected but radiation dominates over convection in the transport of energy. Then the scaling of the stellar mass $M$ with $m_{\mathrm{P}}$ for fixed $T_{c}$ may be obtained for a class of stars (so-called "homologous stars" 4 ) by applying homologous transformations to the equations of hydrodynamical equilibrium. To perform such an analysis, we must phenomenologically model the opacity of the star and the rate of energy generation per unit mass with the respective formulae [56],

$$
\kappa \approx \kappa_{0} \rho^{n} T^{-s}, \quad \epsilon \approx \epsilon_{0} X^{2} \rho T^{\nu} .
$$

The terms $\kappa_{0}$ and $\epsilon_{0}$ are constants while the exponents $n, s$, and $\nu$ depend on the physical properties of the star. The Kramers opacity of intermediate mass stars such as our sun

\footnotetext{
${ }^{4}$ This is a very restrictive class, since by definition the mass distribution for two homologous stars of mass $M_{i}$ and radius $R_{i}$ must satisfy $m_{1}(r) / M_{1}=m_{2}\left(r R_{2} / R_{1}\right) / M_{2}$, where $m_{i}(r)$ is the mass contained within a sphere of radius $r$.
}

is modeled using $n=1$ and $s=7 / 2$. Then it can be shown that, independent of $\nu$, the stellar mass scales like $M \propto$ $X^{1 / 3} m_{\mathrm{P}}^{10 / 3}$ for fixed central temperature [56]. For such stars $T_{s} / T_{c}$ is not constant, so one cannot use Eq. (53). However, at fixed central temperature the homology transformations determine the scaling of the luminosity to be $L \propto$ $X^{2} m_{\mathrm{P}}^{6} M^{-1} \propto X^{5 / 3} m_{\mathrm{P}}^{8 / 3}$. Inserting these scalings into Eq. (51) gives

$$
\hat{\tau}_{\star}^{\prime} \approx \hat{X}^{-1 / 3} m_{\mathrm{P}}^{2 / 3},
$$

where $\hat{\tau}_{\star}^{\prime}$ is the main-sequence lifetime in units of an appropriate lifetime evaluated within our universe.

This gives a slightly weaker dependence on $m_{\mathrm{P}}$ than Eq. (55); however stars that are well described by these approximations have shorter lifetimes than those described by an $i=3 / 2$ polytrope. In addition, the physical characteristics that make these approximations applicable will not continue to describe stars as we decrease $m_{\mathrm{P}}$ with $T_{s}$ fixed. (They do continue to describe stars as $m_{\mathrm{P}}$ is increased for fixed $T_{s}$, since this tends to lessen the importance of convection.) Nevertheless, this confirms the qualitative form of Eq. (55) and suggests that an analogous analysis would apply if surface temperatures closer to that of the sun were demanded.

\section{Heavy element production}

Supernovae are believed to be the exclusive source of heavy elements within our universe. However, the dynamics of supernovae are very complex and are still not fully understood (for reviews see, for example, Refs. [60]). Therefore ensuring the existence of supernovae in universes with differing values of $m_{\mathrm{P}}$ is clearly speculative. In this section we simply provide some qualitative remarks in support of this possibility. There are many types of supernovae within our universe; for convenience we focus on what are called type Ia supernovae.

Type Ia supernovae are understood to erupt via the accretion of matter by a white dwarf star. Meanwhile, white dwarfs are created when a star has consumed all of its hydrogen and helium fuel but does not possess sufficient mass either to drive its central temperature high enough to ignite carbon fusion or to form a black hole. According to the scaling relationships discussed in the previous section, the first condition is always satisfied given that it is satisfied within our universe. On the other hand, the Schwarzschild radius and the physical radius of a star at fixed central temperature both scale as $R \propto m_{\mathrm{P}}^{-2} M$. Therefore white dwarfs will exist in all of the universes that we consider.

The supernova of an accreting white dwarf proceeds when its growing mass reaches the Chandrasekhar limit and the star becomes unstable through the nuclear ignition of its carbon. The relevant physical scales for this phenomena are set by the Chandrasekhar mass and the binding 
energy of a Chandrasekhar mass at about a Schwarzschild radius. Since these and the typical stellar mass all scale as $m_{\mathrm{P}}^{3}$, it seems plausible that type Ia supernovae would occur within universes with significantly differing values of $m_{\mathrm{P}}$. This ensures the production of heavy elements within these universes.

There is a possible caveat to this result. Within the context of another anthropic analysis, it has been remarked [18] that the relatively low production of oxygen by type Ia supernovae may significantly hinder the formation of life if oxygen is not generated elsewhere. However, it is not clear that this suppression, roughly 3\%-8\% relative type II supernova [61], is sufficient to render life overwhelmingly unlikely. The arguments of Ref. [18] were aimed against a scenario where type II supernova would definitely not occur. Since these supernova occur for a wide range of stellar masses within our universe, it is plausible that universes with $m_{\mathrm{P}}$ not too unlike ours will also contain type II supernovae. It is beyond the scope of this paper to investigate more precisely for what values of $m_{\mathrm{P}}$ these supernovae will occur.

\section{G. Stability of stellar systems}

As is illustrated in Refs. [15,19], an important anthropic constraint derives from requiring that stellar systems are stable against cosmic disruptions. Specifically, if a second star grazes too close to an existing stellar system, then a habitable planet may be thrown out of its anthropically fortuitous orbit. Here we seek a constraint to ensure that such encounters are typically too infrequent to interfere with the evolution of life. First, we define a destructive encounter rate,

$$
\gamma \sim n_{\star} \sigma_{\star} v_{p}
$$

where $n_{\star}$ is the number density of stars, $\sigma_{\star}=\pi b^{2}$ is the cross section for an encounter with "fatal" impact parameter $b$, and $v_{p}$ is the typical peculiar velocity of a star. Note that all of the stars within a given neighborhood have the same circular velocity; thus the circular velocity does not contribute to the encounter rate.

The typical peculiar velocity of the stars in a galaxy is approximately determined by the temperature of the constitutive baryons during the phase of star formation. Since the baryons in a galaxy quickly cool to about $T_{\mathrm{H}} \approx 10^{4} \mathrm{~K}$ and cool relatively slowly thereafter, we take this to be the relevant temperature. The corresponding peculiar velocity is then given by Eq. (42). For $\hat{m}_{\mathrm{P}}=1$ this gives a typical peculiar velocity of about $v_{p} \simeq 20 \mathrm{~km} / \mathrm{s}$ which agrees well with observation. The number density of stars $n_{\star}$ is equal to the number density of baryons divided by the typical number of baryons within a star, $N_{\star}$. Recall from Sec. IIE that the number density of baryons within a galactic halo is equal to $f_{b} \rho_{\text {vir }} / m_{N} \mu_{b}$. Therefore $n_{\star}$ can be written

$$
n_{\star}=\frac{f_{\star} f_{b} \rho_{\mathrm{vir}}}{m_{N} \mu_{b} N_{\star}},
$$

where $f_{\star}$ is a fudge factor inserted to account for the increased density of the galactic disk, for the fraction of baryons that do not end up within stars, and for any clustering that may be involved in the star formation process. A typical star within our universe contains $10^{57}$ baryons; therefore the results of Sec. IIF suggest $N_{\star} \sim$ $10^{57} \hat{m}_{\mathrm{P}}^{3} / \hat{\mu}_{\star} \sim 10^{57} \hat{m}_{\mathrm{P}}^{3} / \hat{\mu}_{b}$. To estimate $f_{\star}$ is somewhat more challenging.

The factor $f_{\star}$ accounts for several effects. For example, stars may form in clusters such that most stars exist in a neighborhood of higher density than the average density of stars in a galaxy. On the other hand, a significant fraction of baryons may compose a relatively diffuse interstellar gas and therefore not contribute to the stellar encounter rate. As it is beyond the scope of this paper to compute the $m_{\mathrm{P}}$ dependence of these effects, we simply treat them as being independent of $m_{\mathrm{P}}$. Meanwhile, we also expect $f_{\star}$ to be proportional to the relative density of the galactic disk to that of the baryons in the halo. Rather than concern ourselves with the specific geometry of the galactic disk, we study a simple model to obtain the $m_{\mathrm{P}}$ dependence. We expect the factor $f_{\star}$ to roughly scale like

$$
f_{\star} \propto \frac{R^{3}}{H_{\text {disk }} R_{\text {disk }}^{2}},
$$

where $H_{\text {disk }}$ is the typical disk thickness. This can be written [53]

$$
H_{\mathrm{disk}} \propto m_{\mathrm{P}}^{2} M_{\mathrm{disk}}^{-1} R_{\mathrm{disk}}^{2} v_{p}^{2} .
$$

Meanwhile, to solve for $R_{\text {disk }}$ we note that the circular velocity for stars is given both by Eq. (44) and by,

$$
v_{c} \propto m_{\mathrm{P}}^{-1} M_{\mathrm{disk}}^{1 / 2} R_{\mathrm{disk}}^{-1 / 2} .
$$

Equating these expressions gives $R_{\text {disk }} \propto \lambda^{2} f_{b}^{-1} R$. Finally, putting all of this together gives

$$
f_{\star} \propto \frac{f_{b}^{5}}{\lambda^{8}} \frac{v_{\mathrm{vir}}^{2}}{v_{p}^{2}} .
$$

Note the strong dependence of $f_{\star}$ on the spin parameter $\lambda$. The spin parameter is a stochastic variable with statistical properties related to those of $\zeta_{\text {eq }}$. For example, the Milky Way appears to be characterized by $\lambda \approx 0.06$ [62] while typical galaxies may have $\lambda$ a factor of 2 larger or smaller than this [54]. This and other factors suggest that the factor $f_{\star}$ may vary widely among galactic environments within any particular universe. In addition, as explained above we have ignored several effects that might enter into $f_{\star}$. For concreteness we normalize $f_{\star}$ to the value that describes our solar environment in the Milky Way, $f_{\star} \sim 10^{5}[15]$. This gives 


$$
f_{\star} \sim 10^{5} \hat{m}_{\mathrm{P}}^{-2} \hat{\mu}_{b} \hat{f}_{b}^{5} \mu^{2 / 3} \hat{\rho}_{\mathrm{eq}}^{1 / 3} \hat{\zeta}_{\mathrm{eq}} s(\mu),
$$

where the dependence of $f_{\star}$ on $m_{\mathrm{P}}$ stems entirely from its dependence on $f_{b}$ and $T_{\text {vir }}$.

It is left to calculate the impact parameter for fatal encounters. We are specifically interested in the persistence of stellar systems that contain a planet in orbit about a star such as those considered in Sec. II F. In addition, we focus on planetary orbits that receive electromagnetic radiation with an intensity that is comparable to that from the sun at the orbit of the earth. We then assume that an encounter will not be devastating to such a stellar system if the gravitational field from the grazing star is less than a tenth that of the primary star in the vicinity of the orbiting planet. Therefore we approximate $b$ to be roughly $\sqrt{10}$ times the radius of orbit for a planet receiving about the same stellar intensity as the earth but in orbit about the stars studied in Sec. II F. Note that the luminosity of a star is $L \propto$ $R^{2} T_{s}^{4}$ while the intensity at a distance $r$ is $I \propto L / r^{2}$. Therefore

$$
b=\sqrt{10} r_{\mathrm{au}} \frac{R_{\otimes} T_{\circledast}^{2}}{R_{\odot} T_{\odot}^{2}} \frac{R_{\ominus}}{R_{\circledast}},
$$

where the subscript $\odot$ designates a quantity for the sun, $\otimes$ designates a star with surface temperature $T_{\otimes}=3500 \mathrm{~K}$ in our universe, $R_{\ominus}$ is the radius of a star with this surface temperature within a universe with a different value for $m_{\mathrm{P}}$, and $r_{\mathrm{au}}$ is $1 \mathrm{AU}$. Equation (65) has been written so that every quantity can be evaluated within our universe except for $R_{\ominus} / R_{\otimes} \approx \hat{m}_{\mathrm{P}}$, which is deduced using the results of Sec. IIF.

We put all these results together to obtain $\gamma$. The constraint that stellar systems typically survive a dangerous close encounter for long enough that life may evolve is $\tau_{\text {evol }} \lesssim \gamma^{-1}$, where again $\tau_{\text {evol }}=5 \times 10^{9}$ yrs. In terms of cosmological parameters this is

$$
\hat{m}_{\mathrm{P}}^{3} \hat{\mu}_{b}^{-1 / 2} \hat{f}_{b}^{-6} \hat{\rho}_{\mathrm{eq}}^{-4 / 3} \hat{\zeta}_{\mathrm{eq}}^{-4} \mu^{-2 / 3} s^{-4} \gtrsim 10^{-5},
$$

where we have used the models of Ref. [59] to substitute $R_{\otimes} T_{\otimes}^{2} / R_{\odot} T_{\odot}^{2} \approx \sqrt{L_{\otimes} / L_{\odot}} \approx 0.14$. Decreasing $m_{\mathrm{P}}$ reduces the cross section for dangerous impacts, since the "anthropically favorable" radius decreases, yet increases the number density of stars. The net effect is a constraint against decreasing $m_{\mathrm{P}}$. The explicit constraint implied for $m_{\mathrm{P}}$ is given by

$$
\left(\frac{1}{6} \hat{m}_{\mathrm{P}}^{-\beta}+\frac{5}{6} \hat{m}_{\mathrm{P}}^{-1}\right)^{2 / 3} \hat{m}_{\mathrm{P}}^{3+4 \alpha+6 \beta} \mu^{-2 / 3} s^{-4} \gtrsim 10^{-5},
$$

where $\hat{\mu}_{b}$ depends relatively weakly on $m_{\mathrm{P}}$ and has been ignored. The curves that saturate this inequality are displayed in Fig. 1 using the label " $\gamma^{-1}=\tau_{\text {evol }}$."

\section{H. Summary}

Let us now summarize the results of the previous sections. Many anthropic constraints depend on the primordial curvature perturbation $\zeta_{\text {eq }}$ and on the baryon to photon ratio $\eta$. Lacking any standard model for the generation of either of these, we write them generically as $\zeta_{\text {eq }} \approx \hat{m}_{\mathrm{P}}^{-\alpha}$ and $\eta \approx \hat{m}_{\mathrm{P}}^{-\beta}$, where $\hat{m}_{\mathrm{P}}$ is the ratio between the apparent Planck mass and the value obtained within our universe. For the most popular models of inflation, $\alpha$ ranges between one $\left(m_{\varphi}^{2} \varphi^{2}\right.$ chaotic inflation) and three (hybrid and natural inflation). Meanwhile, popular models of baryogenesis give $\beta$ between zero (efficient leptogenesis and electroweak baryogenesis) and 3/2 (specific models of SUSY Affleck-Dine baryogenesis).

Most of the anthropic constraints under consideration are displayed in Fig. 1, for representative values of $\alpha$ and $\beta$. We assume a WIMP candidate to dominate the dark matter density. Many constraints depend on the total mass within the galactic halo for which they are evaluated. This mass is denoted $\mu$ and is measured in units of the Milky Way mass, or $10^{12}$ solar masses. Note the empty circle in each panel of Fig. 1. This corresponds to a mass scale equal to the mass of the Milky Way with a Planck mass equal to the value obtained within our universe. The region within the $\mu-\hat{m}_{\mathrm{P}}$ plane that is excluded by any constraint is the region that does not include this circle. For clarity we do not display the constraints that primordial black holes form a subdominant contribution to the energy density of the universe and that the dark matter dominates over baryonic matter. These are weaker constraints than those displayed in Fig. 1 and they are easy to calculate from Eqs. (20) and (32). Finally, we note that many of the constraints in Fig. 1 are deduced by assuming that other constraints are satisfied. For example, the "disk inst." curve is changed when the constraint represented by the curve " $t_{\mathrm{rec}}=e^{-2 / 3} t_{\mathrm{vir}}$ " is not satisfied. The continuous curves in Fig. 1 are intended to guide the eye.

We annotate Fig. 1 as follows. A number of constraints come from the various levels of structure formation. The curve labeled " $\tau_{\text {cool }}=\tau_{\text {grav }}$ " (this curve has a distinctive "dorsal fin" shape) marks the separation between the mass scales of halos that contain baryons which cool faster than they (would) collapse and those that do not. As explained in Sec. IIE2, this is one among a set of sufficient, but perhaps not necessary, conditions that allow for galaxy formation. Another one of these conditions is that galactic disks be Jeans-unstable, which occurs below the curve labeled "disk inst." in Fig. 1. Meanwhile, structure formation requires that overdensities separate from the cosmic expansion before the domination of the cosmological constant halts their growth. This requirement is filled below the curve labeled " $\sigma_{\infty}=1.69$." Finally, our analysis of structure formation assumes that galactic halos virialize after recombination, which occurs for $m_{\mathrm{P}}$ values located above the curve labeled " $t_{\text {rec }}=e^{-2 / 3} t_{\text {vir." }}$ " Alternative paths to structure formation are discussed in Appendix A.

We also consider a few anthropic criteria that are not directly related to structure formation. For example, an- 
other constraint that we consider is that galaxies not be so small that they are blown apart by internal supernovae. This will not happen if the binding energy of a galactic halo well exceeds the energy released via supernovae. This condition is satisfied for $\mu$ and $\hat{m}_{\mathrm{P}}$ to the right of the curve labeled " $E_{\text {bind }}=E_{\mathrm{sn}}$." In addition, one might require that collisions between stellar systems be such that impact parameters so small as to dislodge a habitable planet occur on a time scale that is much larger than the evolutionary time scale, here taken to be $\tau_{\text {evol }} \approx 5 \times 10^{9}$ yrs. This constraint is satisfied above the curve labeled " $\gamma^{-1}=\tau_{\text {evol }}$." Finally, one might wish to restrict attention to universes that contain stars that have surface temperatures in excess of about $3500 \mathrm{~K}$ and that have main-sequence lifetimes in excess of about $4 \times 10^{9}$ years. These correspond to positions above the line labeled " $\tau_{\star}=\tau_{\text {evol }}$ " in Fig. 1.

Except for the stellar lifetime constraint, every constraint displayed in Fig. 1 depends on the size of the initial overdensity that eventually grows into a galaxy. The curves in Fig. 1 correspond to choosing this initial fluctuation to be the rms of the density perturbations at a scale $\mu$ evaluated at matter-radiation equality. However, the initial overdensity describing any galaxy is a stochastic variable that may be larger or smaller than this. Therefore all of the curves in Fig. 1 will be shifted when one considers galaxies that are away from the norm. In addition, the disk instability and close encounters curves (labeled "disk inst." and " $\gamma^{-1}=\tau_{\text {evol }}$ ", respectively) depends very strongly on other stochastic quantities, such as the galactic spin parameter (see Sec. II G). Therefore the range of $m_{\mathrm{P}}$ that is consistent with the above constraints is larger than the windows in Fig. 1 would suggest if one allows observers to arise in atypical environments within any given universe.

\section{THE PROBABILITY DISTRIBUTION FOR THE SCALE OF GRAVITY}

If the Planck mass $m_{\mathrm{P}}$ scans across a landscape of universes, then the value within any particular universe may not be uniquely determined. However, with an understanding of the landscape and a calculus to regulate over a conceivably infinite number of infinitely expansive universes, we may in principle calculate the distribution of $m_{\mathrm{P}}$. Since we cannot access any of the other universes within the landscape, such a distribution cannot be directly tested. Nevertheless, we may still use this distribution to calculate the likelihood that we should observe the value of $m_{\mathrm{P}}$ that we do. As we are forced to test this distribution using only our universe, we must be careful to account for any selection effects that would attenuate the distribution of $m_{\mathrm{P}}$.

These selection effects generate a factor $\mathcal{S}$ that multiplies the "prior" distribution $I$. Thus we write the probability to measure the Planck mass to be $m_{\mathrm{P}}$,

$$
P\left(m_{\mathrm{P}}\right)=\mathcal{S}\left(m_{\mathrm{P}}\right) \mathcal{I}\left(m_{\mathrm{P}}\right) .
$$

The factor $I\left(m_{\mathrm{P}}\right)$ may be taken as the likelihood for universes with Planck mass $m_{\mathrm{P}}$ to arise within the multiverse, while $\mathcal{S}\left(m_{\mathrm{P}}\right)$ may be understood as the likelihood for observers to arise within those universes. We restrict our prior $I$ to account for only universes exactly like ours except for their value of $m_{\mathrm{P}}$. This is equivalent to restricting the selection criteria in $\mathcal{S}$. As mentioned in the introduction, there are many subtle issues that complicate the calculation of $\mathcal{S}$ and $I$. Our purpose here is not to resolve any of these issues based on technical grounds. Instead we explore an empirical constraint that may complicate some proposals to address them.

One might expect the likelihood for a universe to support observers to be proportional to the total baryonic mass within galaxies in that universe. (Note that here and below we do not presume proportionality factors to be independent of $m_{\mathrm{P}}$.) Meanwhile, the baryonic mass within galaxies is proportional to the total energy within a universe. This quantity diverges in proportion to the volume of the universe. Nevertheless, we may hope for a regularization scheme that allows for the volumes of universes to be compared. Since the energy density does not redshift during inflation, it is possible that when volumes are properly regulated, the ratio between the total energy densities of two universes will be proportional to the ratio of their inflationary expansion factors.

While this argument may be intuitively appealing, such a prescription for volume-based weighting presents wellknown difficulties [24]. For instance, its conclusion is crucially dependent on a specific global spacelike slicing, which is ambiguous outside the horizon of any one observer. For example, an observer can choose a spacelike slicing that is engineered to create a very large initial volume for the observer's own universe, while also suppressing the initial volume of the universes of casually disconnected observers. The suppression of an initial volume can be used to cancel the inflationary expansion factor, such that this slicing would give a dramatically different counting than the weighting described above.

Nevertheless, in at least one proposal this ambiguity has been overcome and the result includes a selection effect that weights universes according to their inflationary volume (see Garriga et al. (2006) among Refs. [24]). We assume that this result holds and write

$$
\mathcal{S}\left(m_{\mathrm{P}}\right)=\mathcal{A}\left(m_{\mathrm{P}}\right) \mathcal{V}\left(m_{\mathrm{P}}\right) .
$$

Here $\mathcal{A}$ is proportional to the anthropic factor, which ultimately gives the likelihood per unit volume for some class of observer to arise within a universe. Depending on one's notion of an observer, $\mathcal{A}$ might include, for example, the baryonic mass fraction within galaxies, the fraction of stars with lifetimes in excess of few billion years, etc. The factor $\mathcal{V}$ is the inflationary expansion factor for the universe, 


$$
\mathcal{V}\left(m_{\mathrm{P}}\right)=e^{3 N\left(m_{\mathrm{P}}\right)},
$$

where $N\left(m_{\mathrm{P}}\right)$ is the number of e-folds if inflation that typically occurs after a universe with Planck mass $m_{\mathrm{P}}$ has arisen within the multiverse.

If inflation is driven by a single canonical scalar field, then the number of e-folds of inflation is

$$
N=\frac{1}{m_{\mathrm{P}}^{2}} \int_{\varphi_{f}}^{\varphi_{i}} \frac{V}{V_{\varphi}} d \varphi
$$

Here $\varphi_{f}$ is the value of the inflaton when inflation ends, set by when the first slow-roll parameter equals unity, and $\varphi_{i}$ is the value of the inflaton when inflation begins. Note that in general $N$ depends explicitly on $m_{\mathrm{P}}$. As a specific example, consider chaotic inflation with an inflaton potential $V(\varphi)=\frac{1}{2} m_{\varphi}^{2} \varphi^{2}$. This gives

$$
N=\frac{1}{4} \frac{\varphi_{i}^{2}}{m_{\mathrm{P}}^{2}}-\frac{1}{2}
$$

It seems evident that $N$ will generically depend on $m_{\mathrm{P}}$. Yet without understanding the mechanism by which a universe is obtained within the multiverse, it is not clear what is the (typical) value of $\varphi_{i}$ and what is its dependence on $m_{\mathrm{P}}$.

To illustrate that the explicit and implicit dependences of $N$ on $m_{\mathrm{P}}$ are not expected to cancel, three models to determine $\varphi_{i}$ are now considered. The first model sets $\varphi_{i}$ to be the value where classical evolution of $\varphi$ begins to dominate over the quantum fluctuations experienced whenever a mode exits the Hubble radius. This is set by the solution to

$$
\frac{1}{\sqrt{12} \pi} \frac{V^{3 / 2}}{m_{\mathrm{P}}^{3} V_{\varphi}}=1
$$

If this is the case, the total number of e-folds is

$$
N \simeq \sqrt{6} \pi \frac{m_{\mathrm{P}}}{m_{\varphi}} .
$$

The second model assumes that $\varphi_{i}$ is determined by where the inflaton energy density equals the Planck energy density. In this case,

$$
N \simeq \frac{m_{\mathrm{P}}^{2}}{m_{\varphi}^{2}}
$$

On the other hand, if $\varphi_{i}$ is determined by where the inflaton energy density equals $M^{4}$, then

$$
N \simeq \frac{M^{4}}{m_{\varphi}^{2} m_{\mathrm{P}}^{2}} .
$$

Not only does $N$ generally depend on $m_{\mathrm{P}}$, but the dependence is very strong for $m_{\mathrm{P}}$ near the value obtained within our universe. Consider, for example, the case of chaotic inflation with $\varphi_{i}$ set by Eq. (73). Then $N \sim 10^{5} \hat{m}_{\mathrm{P}}$, where $\hat{m}_{\mathrm{P}}$ is the Planck mass in units of the value obtained within our universe. Meanwhile, if Eq. (75) sets the value of $\varphi_{i}$, then $N \sim 10^{10} \hat{m}_{\mathrm{P}}^{2}$. Clearly different choices for $\varphi_{i}$, and, in particular, different models of inflation, will in general give a different dependence of $N$ on $m_{\mathrm{P}}$. However, the dependence is always strong. This is because our universe experienced a large number (at least about 60) of e-folds of inflation.

The ambiguity over the $m_{\mathrm{P}}$ dependence of $N$ is not of concern. The important result is that so long as the dependence on $m_{\mathrm{P}}$ of $\mathcal{A}$ and $I$ is significantly weaker than the strong exponential dependence in $\mathcal{V}$, then we expect $m_{\mathrm{P}}$ to be most probably observed very near one of the boundaries of the anthropic range. The analysis of Sec. II reveals that this is not the case with at least the factor $\mathcal{A}$ and the value of $m_{\mathrm{P}}$ observed within our universe. We illustrate this with an explicit example in Appendix B. There we show that even in the contrived case where $N \sim 60 \hat{m}_{\mathrm{P}}$, the volume factor $\mathcal{V}\left(m_{\mathrm{P}}\right)$ overwhelms what appears to be one of the tightest anthropic constraints. This pushes the expectation value for $m_{\mathrm{P}}$ well beyond what we estimated to be the anthropic boundary, while the value obtained within our universe sits far down the tail of the distribution. This is exactly analogous to the runaway " $\sigma$-problem" and the " $Q$ catastrophe" introduced in Refs. [29]. We refer to our example as the " $m_{\mathrm{P}}$-problem."

The $\sigma$-problem and $Q$ catastrophe were motivated by the fact that in many models of inflation the total number of efolds of inflation depends on the inflationary parameters that also set the level of density perturbations (the authors of Refs. [29] use the notations $\sigma \sim Q \sim \zeta)$. For example, in chaotic inflation with potential $V(\varphi)=\frac{1}{2} m_{\varphi}^{2} \varphi^{2}$ one finds $N \sim \zeta^{-1}$. Therefore if the inflationary parameters may scan over the landscape, by the same argument given above we expect $\zeta$ to be pushed to one of its anthropic boundaries, whereas in our universe it sits comfortably near the middle of the anthropic window [15]. It has been pointed out [63] that this argument is not completely satisfactory, since by hypothesis universes with an enormous number of e-folds are preferred. In such universes, $\zeta$ may plausibly depend on different parameters during a long stretch of early inflation than it does near the end of inflation, when scales important to the formation of structure are generated. Moreover, we note that the curvature perturbation is related to the first slow-roll parameter,

$$
\zeta \sim \frac{1}{\sqrt{\epsilon_{I}}} \frac{V^{1 / 2}}{m_{\mathrm{P}}^{2}} .
$$

Inflation of longer duration requires a smaller $\epsilon_{I}$, yet for inflation to end at all requires that at some point $\epsilon_{I}$ evolve toward unity. Therefore $\zeta_{\text {eq }}$ may be significantly decreased from its value during most of inflation by the necessary 
condition that $\epsilon_{I}$ interpolate between some very small value and unity by the end of inflation.

We emphasize that the $m_{\mathrm{P}}$ problem is not hampered by these issues. That is, unlike $\zeta, m_{\mathrm{P}}$ is a constant within any given universe. ${ }^{5}$ We also emphasize that if the model of inflation that describes our universe exhibits an $m_{\mathrm{P}}$ problem, then allowing more parameters to vary across the landscape cannot mitigate this problem. That is, although allowing more parameters to vary might dramatically shift the expectation value for $m_{\mathrm{P}}$ after the additional parameters have been marginalized, this can only happen if the overwhelming majority of universes near the new expectation value have values for the other parameters that are very different than ours. We would still be left with the challenge to explain why we find ourselves in a universe like ours, and not with these different parameter values.

There are significant caveats to this result. First of all, it is not clear that the selection effects in $\mathcal{S}$ should actually factorize as in Eq. (69). Since the diverging volumes of subuniverses is one of the circumstances that complicates making landscape predictions, we cannot be assured that the resolution of this problem will result in universes with greater inflationary expansion factors being more likely to harbor observers. Another caveat to this discussion is that little is known about the distribution $I\left(m_{\mathrm{P}}\right)$. As we have defined it, this term receives two separate contributions. One contribution comes from the distribution of $m_{\mathrm{P}}$ values over the landscape, that is the frequency of $m_{\mathrm{P}}$ values among the number of metastable states that are allowed by the underlying theory. A second contribution comes from the dynamics of the multiverse, which may prefer certain metastable states over others as the multiverse evolves in time. This is because the tunneling and diffusion rates of quantum fields will in general depend on $m_{\mathrm{P}}$, such that metastable states with certain values of $m_{\mathrm{P}}$ will appear more frequently within the multiverse than others. This $m_{\mathrm{P}}$ dependence within $I\left(m_{\mathrm{P}}\right)$ could be very strong; see, for example, the studies of quantum diffusion in Refs. [23].

Therefore $I\left(m_{\mathrm{P}}\right)$ may depend more sharply on $m_{\mathrm{P}}$ than does $V\left(m_{\mathrm{P}}\right)$, with a local peak within the anthropic range. This might at first seem incredibly fortuitous. However, the situation is very different from the case of the cosmological constant $\rho_{\Lambda}$. In that case we observe $\rho_{\Lambda}$ to be very far from its "natural" value and therefore we must presume a very diverse and densely packed landscape in order for the value that we observe to exist at all. However, since we do not

\footnotetext{
${ }^{5}$ Of course, the inflationary landscape hypothesis presumes that the fields $\phi$ described in the introduction, c.f. Eq. (1), will evolve within the multiverse. Indeed, this is how the landscape is populated. However, we assume that the vacua defined by the fields $\phi$ are selected prior to the period of inflation in which we take interest, during which $m_{\mathrm{P}}$ is constant. Specifically, the factor $I$ is assumed to account for any selection effects due to the field evolution prior to this period of inflation, and the terms $\mathcal{A}$ and $\mathcal{V}$ are defined to apply only after a particular metastable state, with a specific value of $m_{\mathrm{P}}$, has been selected.
}

know the natural value of $m_{\mathrm{P}}$, its landscape window could be much smaller. ${ }^{6}$ In addition, it is possible that the landscape is not as densely populated as we have presumed, in particular, once we restrict attention to metastable states in every way like ours except in the value of $m_{\mathrm{P}}$. For example, if the spacings between allowed values of $m_{\mathrm{P}}$ are significant next to the size of the anthropic window, then our value of $m_{\mathrm{P}}$ might be consistent with the shape of $I\left(m_{\mathrm{P}}\right)$.

Furthermore, the model of inflation that describes our universe may not actually exhibit an $m_{\mathrm{P}}$ problem. This would happen, for example, if the number of e-folds of inflation that describe this model were independent of $m_{\mathrm{P}}$ or had a maximum for some finite value of $m_{\mathrm{P}}$. An interesting example of the latter case occurs when the effective Planck mass is not fixed within our metastable state, but evolves as in Brans-Dicke theory. This scenario has been studied in Refs. [23], where it is shown that for some nonminimally coupled models of inflation, the inflationary expansion factor is maximized when inflation ends at some finite value of $m_{\mathrm{P}}$. Note however that for this or any other model of inflation to avoid the $m_{\mathrm{P}}$ problem, it would have to generate more e-folds of inflation than all of the other anthropically viable possibilities within the landscape. Moreover, the value of $m_{\mathrm{P}}$ that maximizes $N$ would have to lie within the anthropic window.

Finally, it is possible that the analysis of Sec. II missed or underestimated an important anthropic condition. This might appear as the most attractive possibility, but one must be careful to appreciate the strength of the exponential dependence within $V\left(m_{\mathrm{P}}\right)$. In order to cancel this exponential dependence and thus make the observed value of $m_{\mathrm{P}}$ reasonably likely, an anthropic constraint must appear to exponentially suppress the likelihood for observers to arise within our universe. The observed prevalence of galaxies, long-lived stars, supernovae, and planets, along with the observation that our solar system does not seem to occupy a particularly overdense or underdense region within the Milky Way, all seem to suggest that this is not the case. Since there do not yet exist experimentally confirmed theories for inflation, reheating, and baryogenesis, it is still possible that one of these processes presents an anthropic selection effect that provides this exponential suppression. This possibility is explored relative to the reheating temperature and baryogenesis in the context of the $\sigma$-problem in Ref. [64]. Since the reheating tempera-

\footnotetext{
${ }^{6}$ It is tantalizing that within the context of weighting universes by inflationary expansion factors, chaotic inflation with $N$ set by either Eq. (74) or Eq. (75) pushes $m_{\mathrm{P}}$ to larger values, while the largeness of $m_{\mathrm{P}}$ relative to other mass scales is well noted in our universe. Let us assume a fundamental scale $M \sim M_{\mathrm{GUT}}$. Then in this case we expect $m_{\mathrm{P}} \gg M_{\mathrm{GUT}}$, and it is possible that $m_{\mathrm{P}} \sim$ $10^{3} M_{\mathrm{GUT}}$ is simply the largest that the landscape allows. Furthermore, Eq. (74) pushes the inflaton mass $m_{\varphi}$ to smaller values, and perhaps $m_{\varphi}$ is the smallest that the landscape allows. Thus we obtain the apparent hierarchy $m_{\mathrm{P}} \gg M_{\mathrm{GUT}} \gg m_{\varphi}$.
} 
ture in general also depends on $m_{\mathrm{P}}$, this analysis applies equally to our scenario.

\section{ANTHROPIC CONSTRAINTS ON $\Lambda$ AND THE SCALE OF GRAVITY}

It is straightforward to extend the analysis of Sec. II to the case where both $m_{\mathrm{P}}$ and the cosmological constant $\rho_{\Lambda}$ may (independently) scan over a landscape. The only constraint that is affected by this generalization is the requirement that overdensities separate from the Hubble flow before their growth is halted by the domination of the cosmological constant. The maximum amplitude reached by a linear rms overdensity in this scenario is

$$
\sigma_{\infty} \approx 1.44 \times \frac{3}{5} \frac{a_{\Lambda}}{a_{\mathrm{eq}}} \sigma_{\mathrm{eq}} \approx 3.20 \hat{\rho}_{\mathrm{eq}}^{1 / 3} \hat{\rho}_{\Lambda}^{-1 / 3} \hat{\sigma}_{\mathrm{eq}} .
$$

On the other hand, an overdensity has separated from the Hubble flow when a linear analysis gives $\sigma \geq 1.69$ [49]. Therefore a rms fluctuation will eventually form a halo if $\sigma_{\infty} \geq 1.69$, which gives the generalization of Eq. (28):

$$
\hat{\rho}_{\text {eq }} \hat{\sigma}_{\text {eq }}^{3} \hat{\rho}_{\Lambda}^{-1} \gtrsim 0.1 \text {, }
$$

where we find it convenient to henceforth use $\hat{\sigma}_{\text {eq }}$ instead of $\hat{\zeta}_{\mathrm{eq}} s$. This is the only result from Sec. II that changes when $\rho_{\Lambda}$ may scan over the landscape.

Clearly, Eq. (79) is weakened as $\rho_{\Lambda}$ is decreased from the value it obtains within our universe. In this case, Eq. (28) eventually ceases to be the strongest constraint and $m_{\mathrm{P}}$ is bounded from above by one of the other curves in Fig. 1. We may also interpret Eq. (79) as an upper bound on $\rho_{\Lambda}$ for a specified value of $m_{\mathrm{P}}$. In universes with a larger value of $m_{\mathrm{P}}, \rho_{\Lambda}$ is then more tightly bound than in our universe. However, in universes where $m_{\mathrm{P}}$ is smaller than in our universe, the bound on $\rho_{\Lambda}$ may be significantly weakened. This effect can be dramatic. For example, if we take $\hat{m}_{\mathrm{P}}=0.1$ and if $\alpha=1$ and $\beta=0$, then $\rho_{\Lambda}$ may be increased by roughly a factor of $10 \times 10^{6}$ and still satisfy Eq. (79). Of course, to determine the most likely range within which to observe $\rho_{\Lambda}$ requires to determine the prior distribution $I\left(\rho_{\Lambda}, m_{\mathrm{P}}\right)$ and to incorporate all of the selection effects into a factor $\mathcal{S}\left(\rho_{\Lambda}, m_{\mathrm{P}}\right)$, as described in Sec. III. Both of these tasks are beyond the scope of this paper.

Nevertheless, it is worthwhile to proceed but within a very simplified picture. While our level of analysis does not permit even an approximate landscape prediction, our results do imply restrictions on the dependence of $\mathcal{S}$ and $I$ on $m_{\mathrm{P}}$. Our first assumption is that the landscape is so densely packed that we can approximate the prior distribution $I\left(m_{\mathrm{P}}, \rho_{\Lambda}\right)$ to be a continuous and smooth function of both $m_{\mathrm{P}}$ and $\rho_{\Lambda}$. Then we can write the probability distribution for $\rho_{\Lambda}$ in the form

$$
P\left(\rho_{\Lambda}\right) \propto \int \mathcal{S}\left(m_{\mathrm{P}}, \rho_{\Lambda}\right) \mathcal{I}\left(m_{\mathrm{P}}, \rho_{\Lambda}\right) d m_{\mathrm{P}}
$$

We discuss in Sec. III and in Appendix B how our universe appears extremely unlikely to be observed if $\mathcal{S}$ contains a factor proportional to the inflationary expansion factor. Since we wish to expose additional restrictions on $\mathcal{S}$ and $I$, we now assume that $\mathcal{S}$ does not contain this factor.

It is helpful to first consider the distribution $P\left(\rho_{\Lambda}\right)$ when $m_{\mathrm{P}}$ is fixed to the value obtained within our universe. This corresponds to taking $\mathcal{S} \propto \delta\left(\hat{m}_{\mathrm{P}}-1\right)$ and thus eliminating the integral in Eq. (80). References [7] argue that it is appropriate to restrict attention to only positive values of $\rho_{\Lambda}$ and to take the distribution $I$ to be roughly independent of $\rho_{\Lambda}$. Although the rms fluctuation $\sigma_{\text {eq }}$ is constrained by Eq. (79), any particular overdensity may be larger or smaller than $\sigma_{\text {eq. }}$. This implies that galaxies of a given mass will form in universes even when $\rho_{\Lambda}$ is larger than what is allowed by Eq. (79). On the other hand, galaxies of a given mass become statistically rarer as $\rho_{\Lambda}$ is increased. To account for this, it is customary to speculate that the likelihood for a particular universe to be observed is proportional to the fraction of its total mass that collapses into galaxies with masses above some minimum $\mu_{\min }$ [7]. This minimum galaxy mass is presumably set by other anthropic considerations.

The spectrum of density perturbations is at least approximately described by Gaussian statistics. Therefore a randomly selected comoving volume may or may not collapse, depending on the size of the matter overdensity contained within the volume. We parametrize volumes using the mass $\mu$ that they enclose, measured in units of the Milky Way mass, $10^{12} M_{\odot}$. Then the likelihood that a mass $\mu$ will eventually separate from the cosmic expansion is given by the Press-Schechter function [49],

$$
\begin{aligned}
F(\mu) & =\sqrt{\frac{2}{\pi}} \frac{1}{\sigma_{\infty}(\mu)} \int_{1.69}^{\infty} \exp \left[-\frac{1}{2} \frac{z^{2}}{\sigma_{\infty}^{2}(\mu)}\right] d z \\
& =\operatorname{erfc}\left[\frac{0.373 \hat{\rho}_{\Lambda}^{1 / 3}}{\hat{\rho}_{\mathrm{eq}}^{1 / 3} \hat{\sigma}_{\mathrm{eq}}(\mu)}\right] .
\end{aligned}
$$

The percentage of overdensities that eventually virialize is a function of the enclosed mass $\mu$ because the rms amplitude of the initial density perturbations $\sigma_{\text {eq }}$ depends on $\mu$ (see Sec. IIE). The fraction of galaxies that have mass between $\mu$ and $\mu+d \mu$ is $(d F / d \mu) d \mu$. Since $F(\mu \rightarrow$ $\infty)=0$, this means that the fraction of mass contained within galaxies with mass above the mass scale $\mu$ is simply the Press-Schechter function $F$ evaluated at $\mu$. When only the cosmological constant scans over the landscape, $\hat{\rho}_{\text {eq }}=$ $\hat{\sigma}_{\text {eq }}=1$. This gives $P\left(\rho_{\Lambda}\right) \propto \mathcal{S}\left(\rho_{\Lambda}\right) \propto F\left(\mu_{\min }, \rho_{\Lambda}\right)[7,15]$.

In order to study the scenario where both $\rho_{\Lambda}$ and $m_{\mathrm{P}}$ scan over the landscape, we adopt a very simplified picture. First, we assume that $I$ is independent of both $\rho_{\Lambda}$ and $m_{\mathrm{P}}$ over the anthropically allowed window. We emphasize that, unlike the case with $\rho_{\Lambda}$, we are unaware of any physical justification for this assumption regarding $m_{\mathrm{P}}$. 
Second, we restrict our attention to galaxies with masses near the mass of the Milky Way. We perform this restriction simply so that we may ignore the scale dependence of anthropic constraints. It turns out that values of $m_{\mathrm{P}}$ somewhat larger than our own do not contribute significantly toward $P\left(\rho_{\Lambda}\right)$. To highlight this result we simply neglect all constraints on increasing $m_{\mathrm{P}}$. On the other hand, the selection effects that bound $m_{\mathrm{P}}$ from below are very important when determining $P\left(\rho_{\Lambda}\right)$. For simplicity we consider selection effects from only one additional constraint; which is that stellar encounters are rare enough on average to allow for life to evolve in the intervening time. According to Fig. 1, this is usually the strongest constraint on decreasing $m_{\mathrm{P}}$. The exception appears to be the case of low $\alpha$ and low $\beta$, where the galactic cooling constraint can interfere and the stellar lifetime constraint is not far below the close encounters constraint. We simply ignore the cooling constraint and note that we could just as well evaluate $P\left(\rho_{\Lambda}\right)$ for galaxy masses somewhat below the mass of the Milky Way to obtain a similar result. To account for stellar lifetimes, we impose a hard cutoff below $\hat{m}_{\mathrm{P}}=0.1$.

As mentioned above, the mass fraction within galaxies with masses between $\mu$ and $\mu+d \mu$ is $\delta F \equiv$ $(d F / d \mu) d \mu$. This quantity in general depends on the time at which one looks at the universe. We count galaxies in the infinite future, which is practically equivalent to counting galaxies at any time after the domination of $\rho_{\Lambda}$. Then

$$
\frac{d F}{d \mu} \propto \frac{1}{\sigma_{\infty}^{2}}\left|\frac{d \sigma_{\infty}}{d \mu}\right| e^{-1.43 / \sigma_{\infty}^{2}} .
$$

The only $\mu$ dependence within $F$ stems from the dependence on $\sigma_{\infty} \propto \sigma_{\text {eq }} \propto s(\mu)$, where $s(\mu)$ is given by Eq. (23). Within any given universe, to consider only galaxies with a particular mass $\mu$ in the far future is equivalent to selecting only overdensities with a particular amplitude at equality. This is because within that universe overdensities with smaller amplitudes will form galaxies with smaller mass while overdensities with larger amplitudes will form galaxies with larger mass (recall that we look at the universe after $\rho_{\Lambda}$ domination when the growth in overdensities has halted). The amplitude of the initial overdensity that is selected by looking at a particular galaxy mass $\mu$ is the one that gives $\sigma(\mu)=1.69$ in the infinite future.

We must now account for the close encounter constraint mentioned above. This constraint is converted into a selection effect by noting that if the rate of disastrous encounters between stellar systems is $\gamma$, then the probability that a stellar system will survive for a time $\tau$ is $e^{-\gamma \tau}$. The rate $\gamma$ is discussed in Sec. II G. Note that it depends on the amplitude of the initial overdensity that seeded the galaxy. We restrict our attention to galaxies with masses near to the mass of the Milky Way. As described above, these galaxies only come from overdensities that satisfy $\sigma(\mu \approx 1)=$ 1.69 in the infinite future. At equality, these overdensities have an amplitude

$$
\sigma \approx 5 \times 10^{-4} \hat{\rho}_{\Lambda}^{1 / 3} \hat{\rho}_{\mathrm{eq}}^{-1 / 3} .
$$

Now we can write the likelihood $P_{\text {ss }}$ that a stellar system will survive for at least a time $\tau$ within this set of galaxies. We take $\tau=\tau_{\text {evol }} \approx 5 \times 10^{9} \mathrm{yrs}$, which gives

$$
P_{\mathrm{ss}} \approx \exp \left(-7 \times 10^{-7} \hat{m}_{\mathrm{P}}^{-3} \hat{\mu}_{b}^{1 / 2} \hat{f}_{b}^{6} \hat{\rho}_{\Lambda}^{4 / 3} \mu^{2 / 3}\right),
$$

where the dependence on a general mass scale $\mu$ has been restored for future reference.

So far our assumptions correspond to weighting universes by the fraction of stellar systems that survive close encounters for longer than $\tau_{\text {evol }}$ and that exist in galaxies with mass near to the Milky Way mass. We also require $m_{\mathrm{P}} \geq 0.1$ in order to ensure that sufficiently long-lived stars exist in these universes. Finally, we should account for the fact that the abundance of baryons relative dark matter will depend on the value of $m_{\mathrm{P}}$ within each universe. Putting all of this together gives the probability density

$$
\begin{aligned}
P\left(\hat{\rho}_{\Lambda}\right) \propto & \int_{\mu_{\min }}^{\mu_{\max }} d \mu \int_{0.1}^{\infty} d m_{\mathrm{P}} f_{b}\left(m_{\mathrm{P}}\right) P_{\mathrm{ss}}\left(m_{\mathrm{P}}, \hat{\rho}_{\Lambda}, \mu\right) \\
& \times \frac{d}{d \mu} F\left(m_{\mathrm{P}}, \hat{\rho}_{\Lambda}, \mu\right) .
\end{aligned}
$$

The full $m_{\mathrm{P}}$ dependence of $f_{b}, P_{\mathrm{ss}}$, and $d F / d \mu$ is found by substitution of the results from Sec. II. In general Eq. (85) is integrated over a window $\mu_{\min } \leq \mu \leq \mu_{\max }$, but as motivated above, in our main analysis we restrict to a narrow window about $\mu=1$. Finally, as explained previously, Eq. (85) makes the simplifying but unrealistic assumption that $I\left(m_{\mathrm{P}}, \rho_{\Lambda}\right) \approx$ constant.

The results of a numerical computation of $P\left(\rho_{\Lambda}\right)$ are displayed in Fig. 2. For reference, we also display the result when $m_{\mathrm{P}}$ is fixed to the value obtained within our universe (note that this corresponds to $d F /\left.d \mu\right|_{\mu=1}$ and not $F(\mu=$ 1)). Our value of $\rho_{\Lambda}$ corresponds to the origin on this graph. Evidently the assumptions of this section render the observation of $\rho_{\Lambda}$ at or below our value very unlikely. In fact, the fraction of $P\left(\rho_{\Lambda}\right)$ that sits below $\hat{\rho}_{\Lambda}=1$ is about $7 \times 10^{-5}$ for $\alpha=1, \beta=0$ and about $4 \times 10^{-4}$ for $\alpha=3, \beta=3 / 2$. Since relatively large values of $\rho_{\Lambda}$ receive significant weight only when $m_{\mathrm{P}}$ is relatively small, we also see that most of the weight of these distributions comes from values of $m_{\mathrm{P}}$ that are below the value obtained within our universe. This is evidence of a sort of "statistical pressure" that gives greater weight to those values of marginalized parameters that permit a larger value of $\rho_{\Lambda}$. This is why it was unimportant to account for selection effects that constrain $m_{\mathrm{P}}$ from above.

Here we note the importance of the close encounters constraint and of recombination timing constraint (i.e. the " $t_{\mathrm{rec}}=e^{-2 / 3} t_{\mathrm{vir}}$ " constraint) in bounding the anthropi- 


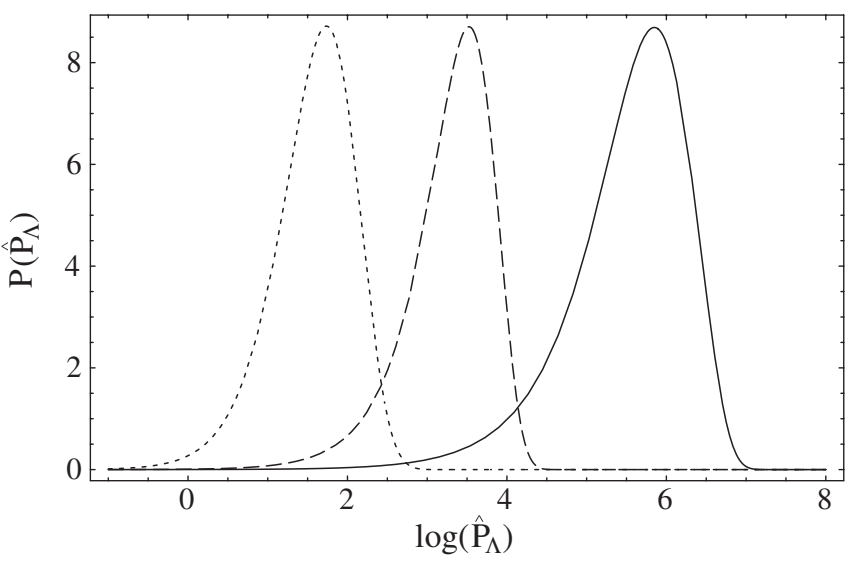

FIG. 2. The distribution $P\left(\hat{\rho}_{\Lambda}\right)$ displayed against $\log \left(\hat{\rho}_{\Lambda}\right)$. The solid curve is $P\left(\hat{\rho}_{\Lambda}\right)$ marginalized over universes in which $m_{\mathrm{P}}$ may vary and with $\alpha=1$ and $\beta=0$, the longer-dashed curve is the same quantity but for $\alpha=3$ and $\beta=3 / 2$, while the shorterdashed curve is $P\left(\hat{\rho}_{\Lambda}\right)$ evaluated when $m_{\mathrm{P}}$ is fixed to the value within our universe. All three distributions are for fixed galactic masses $\mu=1$. See the text for results obtained for a range of galactic masses. The normalizations are chosen for clarity.

cally allowed variation of $\hat{\rho}_{\Lambda}$. Inspecting Fig. 1 indicates that of the constraints limiting $m_{\mathrm{P}}$ from below, the close encounters bound is the strongest. For $\mu=1$, it bounds $\hat{m}_{\mathrm{P}} \gtrsim 10^{-0.2}$ for $\alpha=3$ and $\beta=3 / 2$, and approximately $\hat{m}_{\mathrm{P}} \gtrsim 10^{-0.8}$ for $\alpha=1, \beta=0$. Inserting these limits on $\hat{m}_{\mathrm{P}}$ into Eq. (79) gives maximum values for $\hat{\rho}_{\Lambda}$ in good agreement with the peaks in Fig. 2. Had only the star lifetime constraint $\hat{m}_{\mathrm{P}} \gtrsim 0.1$ been imposed, the maximum allowed value of $\hat{\rho}_{\Lambda}$ would have been much larger. For $\alpha=1$ and $\beta=0, \hat{\rho}_{\Lambda}$ could be as large as $4 \times 10^{7}$. In the case of $\alpha=3$ and $\beta=3 / 2, \hat{\rho}_{\Lambda}$ can be as large as $2 \times$ $10^{14}$. But the anthropic constraints in Fig. 1 for this latter model indicate that the " $t_{\text {rec }}=e^{-2 / 3} t_{\text {vir }}$ " constraint is stronger than the star lifetime constraint. Imposing the recombination constraint requires instead that $\hat{\rho}_{\Lambda}$ be no larger than about $4 \times 10^{8}$.

The analysis that leads to the curves in Fig. 2 gives at best a crude approximation for the actual probability distribution for $\rho_{\Lambda}$. One improvement to the analysis would be to weight universes by the mass fraction that collapses into galaxies that have a range of anthropically favorable masses, instead of the fraction that collapses into only galaxies with the Milky Way mass. Including galaxies with greater masses will tend to push the weight of the distributions toward smaller $\rho_{\Lambda}$, while including galaxies with smaller masses pushes the weight of the distributions toward larger $\rho_{\Lambda}$. We have checked that under the assumptions outlined above, allowing for a range of galaxy masses $0.1 \leq \mu \leq 10$ tends to push the weight of the distributions $P\left(\rho_{\Lambda}\right)$ to slightly larger values of $\rho_{\Lambda}$.

Previous calculations of the distribution $P\left(\rho_{\Lambda}\right)$ integrate over all galaxy masses equal to or larger than the Milky Way mass. Although our anthropic considerations offer no reason to ignore galaxies with mass below that of the Milky Way, and galactic cooling constraints limit the formation of galaxies with larger masses, we nevertheless consider the evaluation of Eq. (85) for a range of masses $1 \leq \mu \leq \infty$. Integrating over $0 \leq \hat{\rho}_{\Lambda} \leq 1$ gives the probability $P_{*}$ that observers in such galaxies would observe a cosmological constant less than or equal to our own. Numerically, we find $P_{*}=1 \times 10^{-3}$ for $\alpha=3, \beta=$ $3 / 2$ and $P_{*}=2 \times 10^{-4}$ for $\alpha=1, \beta=0$. For comparison, we find that in our universe $P_{*}=0.06$. To reiterate, these calculations ignored any additional selection effects that might depend on $\mu$, such as the effects of different galactic cooling rates.

Another improvement to the analysis would be to include more $m_{\mathrm{P}}$-dependent selection effects. The abundance of heavy elements and of long-lived stars with appropriate surface temperatures both seem important when determining the likelihood for observers to arise within a universe. However, the analysis of Sec. II does not shed light on how to calculate these selection effects. One thing that is clear is that the range of typical stellar masses scales as $m_{\mathrm{P}}^{3}$. This means that galaxies of a fixed mass will contain more stars as $m_{\mathrm{P}}$ is decreased. If all else were equal, as $m_{\mathrm{P}}$ is decreased this would result in a greater number of observers per unit baryon mass in a galaxy, which would tend to push the weight of $P\left(\rho_{\Lambda}\right)$ toward larger values of $\rho_{\Lambda}$. In addition, the rate of destructive encounters $\gamma$ is a function of stochastic variables, including, for example, the spin parameter $\lambda$. The statistical distribution of these variables could tend to strengthen or weaken the close encounter constraint as a function of $m_{\mathrm{P}}$. However, when everything else is equal the "statistical pressure" alluded to below Eq. (85) tends to give greater weight to those values of stochastic variables that allow for a smaller $m_{\mathrm{P}}$ and larger $\rho_{\Lambda}{ }^{7}$

\footnotetext{
${ }^{7}$ We have confirmed this phenomena with the following simple example. The close encounter rate $\gamma$ is proportional to a factor $f_{\star}$ that accounts for the increased density of the galactic disk relative to the dark matter halo (see Sec. IIE3). This factor depends sensitively on $\lambda, f_{\star} \propto \lambda^{-8}$. In the preceding analysis, we normalized the factor $f_{\star}$ so as to give the correct stellar density within our neighborhood of the Milky Way. However, $\lambda$ is a stochastic variable. $N$-body simulations suggest that the distribution for $\lambda$ can be approximated using [65]

$$
P_{\lambda}(\lambda) d \lambda \propto \frac{d \lambda}{\lambda} \exp \left[-2 \ln ^{2}(28.6 \lambda)\right] .
$$

This distribution has a peak at about $\lambda \approx 0.03$, while the Milky Way appears to be described by $\lambda \approx 0.06$ [62]. This implies that typical values of $\lambda$ more tightly constrain $m_{\mathrm{P}}$ than the value represented in Fig. 2. Therefore, one might expect that when we treat $\lambda$ as a stochastic variable with distribution $P_{\lambda}(\lambda)$, that the weight of the distributions $P\left(\rho_{\Lambda}\right)$ will shift to smaller values of $\rho_{\Lambda}$. In fact, the opposite trend occurs, as the previously mentioned "statistical pressure" is such that the weight of the distributions $P\left(\rho_{\Lambda}\right)$ actually shifts the location (in $\hat{\rho}_{\Lambda}$ ) of the peak by approximately an order of magnitude toward larger values of $\rho_{\Lambda}$.
} 
Of course, a proper calculation of $P\left(\rho_{\Lambda}\right)$ requires an understanding of the prior distribution for $m_{\mathrm{P}}, I\left(m_{\mathrm{P}}\right)$. It must be emphasized that the shape of $I\left(m_{\mathrm{P}}\right)$ could dramatically influence the shape of the distribution $P\left(\rho_{\Lambda}\right)$. Therefore the results of this section are best understood as an empirical restriction on the dependence of $I\left(m_{\mathrm{P}}\right)$ on $m_{\mathrm{P}}$. Since this is our main point in this section, let us be very explicit. The curves displayed in Fig. 2 suggest that within a very simplified landscape picture, it is very unlikely to observe a value of $\rho_{\Lambda}$ that is at or below the value within our universe. This means that if a landscape picture is to describe our universe, it should contain important ingredients that were neglected in our analysis. In addition, these additional ingredients should provide a strong emphasis for larger values of $m_{\mathrm{P}}$. Thus we conclude that for a landscape picture to describe our universe as among those that are likely to be observed, it is necessary that $I\left(m_{\mathrm{P}}\right)$ or some other neglected selection effect must receive the vast majority of its weight for values of $m_{\mathrm{P}}$ that are very near to or larger than the value obtained within our universe. We emphasize that the analysis of this section did not assume that the inflationary expansion factor enters into landscape calculations.

\section{CONCLUSIONS}

If the magnitude of the apparent Planck mass $m_{\mathrm{P}}$ may scan across a landscape of possibilities, then there may exist universes with physical parameters and interactions in every way like those within our universe except for their value of $m_{\mathrm{P}}$. We have calculated the range over which $m_{\mathrm{P}}$ may scan over such universes while still satisfying a number of anthropic constraints. Perhaps not surprisingly, if we combine all of the anthropic constraints we find a rather narrow window for allowed $m_{\mathrm{P}}$. The results for WIMP dark matter and representative models of inflation and baryogenesis are displayed in Fig. 1. Of course, the window for allowed $m_{\mathrm{P}}$ is expanded if one loosens the anthropic criteria.

More interestingly, this window will expand if an important cosmological quantity is determined by a stochastic process. For example, many scenarios to generate a primordial curvature perturbation depend on the local vev of a light scalar field, as does the density of dark matter when it is determined by the axion. If these models apply, then the curvature perturbation and/or dark matter density are not correlated with changes in $m_{\mathrm{P}}$, and a much larger window for $m_{\mathrm{P}}$ may be able to satisfy anthropic constraints. Our purpose has been to calculate a minimal window for allowed $m_{\mathrm{P}}$, so we have not considered these possibilities in detail.

Even a very small window for allowed values of $m_{\mathrm{P}}$ has important implications for the landscape paradigm. In particular, the probability to observe a particular value of $m_{\mathrm{P}}$ may be weighted by the inflationary expansion factor of universes that contain that value. This effect inputs a strong exponential dependence on $m_{\mathrm{P}}$ into the probability distribution, which must be offset by another strong selection effect near the peak of the distribution. This other selection effect could be a very sharp peak or boundary to the underlying landscape distribution; otherwise the effect must come from an exponentially strong anthropic dependence on $m_{\mathrm{P}}$. Such a strong anthropic dependence on $m_{\mathrm{P}}$ would be in conflict with the observation that $m_{\mathrm{P}}$ has even a narrow anthropic window in our universe. This is another example of the runaway inflation problem discussed in the recent literature.

We also consider the anthropic window for the cosmological constant $\Lambda$ when both $\Lambda$ and $m_{\mathrm{P}}$ are allowed to independently scan over the landscape. Even when the allowed range for $m_{\mathrm{P}}$ is relatively narrow, it still allows for a significant broadening of the allowed range for $\Lambda$. This is because $\Lambda$ is only constrained by the necessity that cosmic structures separate from the Hubble flow before $\Lambda$ domination. Meanwhile, the time at which structures separate from the cosmic expansion is proportional to a high power of $m_{\mathrm{P}}$. The result is that even for values of $m_{\mathrm{P}}$ within the small allowed windows of Fig. 1, $\Lambda$ may be over $10 \times$ $10^{6}$ times larger in other universes than it is within ours. Just because $\Lambda$ may be larger does not automatically imply that our value of $\Lambda$ is less likely to be observed, since selection effects may ultimately weight smaller values of $\Lambda$ more than larger values. We perform a very basic calculation which suggests that anthropic selection effects tend to make larger values of $\Lambda$ more likely to be observed. This suggests that the observation of a cosmological constant at or below the level obtained within our universe is very unlikely unless unknown anthropic selection effects or the underlying landscape distribution of $m_{\mathrm{P}}$ is dominated by values very near to or larger than the value obtained within our universe.

\section{ACKNOWLEDGMENTS}

The authors thank Stearl Phinney, Jonathan Pritchard, Scott Thomas, and Andrei Linde for helpful discussions. This work was supported by the U.S. Department of Energy under contracts No. DE-FG03-92ER40689 and No. DE-FG03-92ER40701.

\section{APPENDIX A: NONSTANDARD PATHS TOWARD STRUCTURE FORMATION}

One might wonder what are the constraints on structure formation if we do not assume that dark matter dominates over baryonic matter, or that virialization occurs after recombination. If dark matter does not dominate over baryonic matter, then the evolution of overdensities in the dark matter does not significantly affect the evolution of overdensities in baryons. Without appreciable dark matter potential wells, baryon overdensities do not grow (even logarithmically) until after recombination. This is because 
in the era before recombination, the Jeans length for the tightly coupled baryons,

$$
R_{J}=\sqrt{\frac{8}{3}} \pi H^{-1} v_{s},
$$

where $v_{s}=1 / \sqrt{3}$ is the speed of sound prior to recombination, is always larger than the Hubble radius. Growth therefore does not occur in either the radiation or baryondominated era until after recombination. Between recombination and the domination of cosmological constant the evolution of overdensities may be approximated by

$$
\sigma \approx\left(a / a_{\text {rec }}\right) \sigma_{\text {rec }} .
$$

The spectrum of fluctuations at recombination $\sigma_{\text {rec }}$ is scale dependent in the sense that it is constant for scales larger than the Hubble radius at recombination but rapidly decreases to zero as one looks at smaller distance scales. This is because of the tight coupling between baryon and radiation overdensities, and because the latter decay after they enter the Hubble radius.

As in the standard picture, after the domination of cosmological constant overdensities will grow by a factor of 1.44 and then stop. Thus the maximum amplitude achieved by a linear analysis of a rms fluctuation is

$$
\sigma_{\infty} \approx 1.44 \times\left(a_{\Lambda} / a_{\mathrm{rec}}\right) \sigma_{\mathrm{rec}} \approx 5 \times 10^{-2} \hat{\rho}_{\mathrm{eq}}^{1 / 3} \hat{\zeta}_{\mathrm{eq}} .
$$

Here we have used that $\sigma_{\text {rec }} \approx 5 \times 10^{-5} \hat{\zeta}_{\text {eq }}$ on scales larger than the Hubble radius at recombination, and that recombination occurs at a temperature $T_{\text {rec }} \approx 3000 \mathrm{~K}$, where we ignore the logarithmic dependence of $T_{\text {rec }}$ on $m_{\mathrm{P}}$ and $\eta$. The formation of structure still requires that a linear analysis gives $\sigma_{\infty} \geq 1.69$ before the growth in overdensities is halted by the domination of cosmological constant. This gives the constraint

$$
\hat{\rho}_{\text {rec }} \hat{\zeta}_{\mathrm{eq}}^{3} \gtrsim 5 \times 10^{5} .
$$

Equation (A4) constrains $m_{\mathrm{P}}$ according to

$$
\left(\frac{1}{6} \hat{m}_{\mathrm{P}}^{-\beta}+\frac{5}{6} \hat{m}_{\mathrm{P}}^{-1}\right) \hat{m}_{\mathrm{P}}^{-3 \alpha} \gtrsim 5 \times 10^{5} .
$$

This constraint is much stronger than the constraint it replaces, Eq. (29).

Allowing for baryons to dominate the matter density of the universe may affect the other constraints in Fig. 1 in two ways. First, the halo density and background density at virialization, $\rho_{\text {vir }}$ and $\rho_{*}$, are now reduced by a factor of $3 \times 10^{-6}$ due to the difference between $\sigma_{\text {rec }}$ and $\sigma_{\text {eq }}$. Second, structure formation only occurs on scales greater than the Hubble radius at recombination, since subhorizon perturbations are suppressed. Ignoring the $m_{\mathrm{P}}$ dependence in $T_{\text {rec }}$, this implies a minimum halo mass set by the horizon mass at recombination, corresponding to a scale of roughly $\mu_{\min } \sim 10^{6}$. It can be shown that no value of $m_{\mathrm{P}}$ satisfies all of the constraints displayed in Fig. 1 after these effects have been included. Dropping the constraint that virialization precede recombination does not change this result.

We now turn to the second assumption of Sec. IIE, which is that recombination occurs at least an e-fold of expansion before virialization. To investigate what happens when virialization occurs before recombination, we adopt the following simplified picture. Dark matter overdensities grow when they enter the Hubble radius, and we assume that they become nonlinear and virialize as they would within our universe. However, growth in the baryon overdensities is hampered by their interaction with the photon Hubble flow before recombination. Therefore we approximate that baryons do not participate at all in the overdensities of the dark matter and are rarefied relative the halo density as they follow the Hubble flow.

Within this simplified model, the final baryon fraction within a halo will be at most about $f_{b} / 18 \pi^{2}$, and will decrease by a factor of $e^{-3} \approx 0.05$ for each e-fold of expansion between virialization and recombination. However, it turns out that only two of the constraints that we consider depend significantly on the baryon fraction of the halo. These are the disk instability constraint of Sec. IIE 3 and the close encounters constraint of Sec. IIG. (The explicit $f_{b}$ dependence in the galactic cooling constraint of Sec. IIE 2 is canceled by an implicit dependence within $\Lambda_{c}$.) To explore whether this situation opens a new window for allowed values of $m_{\mathrm{P}}$, it is helpful to adopt the following picture. Instead of simply eliminating the recombination timing constraint of Eq. (31), we continuously weaken it. For example, we may demand that recombination occur at most $N_{\text {rec }}$ e-folds of expansion after virialization and then study the above constraints as $N_{\text {rec }}$ is increased.

When we do this, we find that the curves in Fig. 1 corresponding to the disk instability constraint, the close encounters constraint, and the recombination timing constraint all slide downward as $N_{\text {rec }}$ is increased. This shifts the allowed window for $m_{\mathrm{P}}$ such that larger values of $m_{\mathrm{P}}$, including the value obtained within our universe, become excluded as lower values become allowed. It turns out that the disk instability curve slides downward at a faster rate than that of the recombination timing curve, so that as the window for allowed $m_{\mathrm{P}}$ moves to smaller $m_{\mathrm{P}}$ it also grows smaller. Ultimately, the window gets pushed against other constraints, such as the stellar lifetime constraint or the galactic cooling constraint, and disappears. This happens at about $N_{\text {rec }} \approx$ a few.

\section{APPENDIX B: ANALYSIS OF A STRUCTURE FORMATION CONSTRAINT}

In Sec. III it is argued that if the probability to observe a particular value of $m_{\mathrm{P}}$ is weighted in part by the inflationary expansion factor of universes that contain that value of $m_{\mathrm{P}}$, then it is overwhelmingly preferred that $m_{\mathrm{P}}$ should be measured at one of the boundaries of its an- 
thropic window. It is clear from the discussion of Sec. II that our value of $m_{\mathrm{P}}$ is not at either of its anthropic boundaries. Nevertheless, it is worthwhile to investigate more quantitatively just how "far" is our value of $m_{\mathrm{P}}$ from its anthropic boundaries. For simplicity we investigate the selection effect from only one anthropic constraint. Specifically, we look at the structure formation requirement that halos virialize before the domination of cosmological constant (Sec. IIE 1). Note that this provides the tightest constraint on $m_{\mathrm{P}}$ according to the curves in Fig. 1.

The arguments of Sec. III are appropriate primarily when the landscape is so densely packed that we can approximate the prior distribution $I\left(m_{\mathrm{P}}\right)$ to be a continuous function of $m_{\mathrm{P}}$ within the anthropic window. The probability to observe $m_{\mathrm{P}}$ to lie within the range $d m_{\mathrm{P}}$ can then be written,

$$
P\left(m_{\mathrm{P}}\right) d m_{\mathrm{P}} \propto \mathcal{A}\left(m_{\mathrm{P}}\right) \mathcal{V}\left(m_{\mathrm{P}}\right) I\left(m_{\mathrm{P}}\right) d m_{\mathrm{P}},
$$

where the factors on the right-hand side are defined in Sec. III. Of course, we are assuming that universes are weighted in part by their inflationary expansion factor $\mathcal{V}$. Since we have no knowledge about the shape of $\mathcal{I}\left(m_{\mathrm{P}}\right)$, we take $I\left(m_{\mathrm{P}}\right) \approx$ constant. As suggested above, we take the anthropic factor $\mathcal{A}$ to be conditioned by only the constraint that halos virialize before the domination of cosmological constant prevents this.

To proceed, we assume that the likelihood to observe a given value of $m_{\mathrm{P}}$ is proportional to the baryon fraction within galaxies of mass greater than or equal to the mass of the Milky Way, $10^{12} M_{\odot}$. Allowing for smaller galaxies or allowing for observers that do not require a galactic environment can only expand the window of allowed $m_{\mathrm{P}}$. The Press-Schechter function [49] gives the fraction of matter that collapses into a galaxy of mass greater than or equal to a given scale. It is derived in Sec. IV and given by Eq. (81). We reproduce it here for convenience,

$$
F=\operatorname{erfc}\left[0.373\left(\frac{\hat{\rho}_{\Lambda}}{\hat{\rho}_{\text {eq }} \hat{\sigma}_{\text {eq }}^{3}}\right)^{1 / 3}\right] .
$$

The prefactor comes in part from evaluating $F$ in the infinite future and at the Milky Way mass scale. The $m_{\mathrm{P}}$ dependence is given by

$$
\left(\frac{\hat{\rho}_{\Lambda}}{\hat{\rho}_{\mathrm{eq}} \hat{\sigma}_{\mathrm{eq}}^{3}}\right)^{1 / 3}=\left(\frac{1}{6} \hat{m}_{\mathrm{P}}^{-\beta}+\frac{5}{6} \hat{m}_{\mathrm{P}}^{-1}\right)^{-4 / 3} \hat{m}_{\mathrm{P}}^{\alpha}
$$

Note that when $\alpha$ and $\beta$ are positive, $F$ is a decreasing function of increasing $m_{\mathrm{P}}$. We are interested in the baryonic matter within galaxies. Therefore $\mathcal{A}$ should contain a factor of the baryon fraction $f_{b}$, given by Eq. (18), along with $F$.

Finally, we take $\mathcal{V}\left(m_{\mathrm{P}}\right) \propto e^{3 N}$ for $N$ e-folds of inflation. We require that $N\left(m_{\mathrm{P}}\right)$ be an increasing function of $m_{\mathrm{P}}$ so that $m_{\mathrm{P}}$ is pushed to larger values, saturating the constraint in Eq. (29). In addition, we want $\alpha=3$ and $\beta=3 / 2$ so that this constraint on increasing $m_{\mathrm{P}}$ is as strong as pos- sible. Rather than propose a specific model of inflation, we assume that one can contrive a model with the relatively weak dependence $N \approx 60 \hat{m}_{\mathrm{P}}$. Then putting all of our assumptions together gives the probability distribution

$$
P\left(m_{\mathrm{P}}\right)=\mathcal{N} f_{b}\left(m_{\mathrm{P}}\right) F\left(m_{\mathrm{P}}\right) e^{3 N\left(m_{\mathrm{P}}\right)},
$$

where $\mathcal{N}$ is a normalization factor. The normalized distribution $P\left(m_{\mathrm{P}}\right)$ is displayed in Fig. 3 for $\alpha=3$ and $\beta=$ $3 / 2$. Although it is intriguing that in this scenario the erfc function overcomes the exponential volume factor, this happens for a value of $m_{\mathrm{P}}$ somewhat larger than the value that we observe. This $\mathcal{O}(1)$ change in $m_{\mathrm{P}}$ is significant due to the exponential sensitivity of $P$ on $m_{\mathrm{P}}$. Indeed, the fraction of the distribution function $P\left(m_{\mathrm{P}}\right)$ that sits below $\hat{m}_{\mathrm{P}}=1$ is completely negligible compared to that which sits above (explicitly, this fraction is roughly $10^{-53}$ ). This distribution is so sharply peaked because $N$ is relatively large. For example, expanding about the local maximum gives $P \sim \exp \left[-c N \Delta^{2}\right]$ where $c \sim \mathcal{O}(1)$ and $\Delta$ is the difference between $\hat{m}_{\mathrm{P}}$ and its value at the maximum. Even if the number of e-folds depends very weakly on $m_{\mathrm{P}}$, for example $N \approx 60 \ln \left(\hat{m}_{\mathrm{P}}\right)$, we still find the preference for larger $m_{\mathrm{P}}$ to be overwhelming.

We now provide a final point of clarification. A careful reader may notice that according to Fig. 3, the values of $m_{\mathrm{P}}$ that are most likely to be observed lie well outside the anthropically allowed windows of Fig. 1. This is because the relevant curves in Fig. 1 are calculated by assuming that all overdensities have initial amplitudes equal to the rms amplitude. Meanwhile, Fig. 3 takes into account that the initial amplitude of an overdensity is at least approximately a Gaussian random variable. The discrepancy between the results in Figs. 1 and 3 reflect that under the

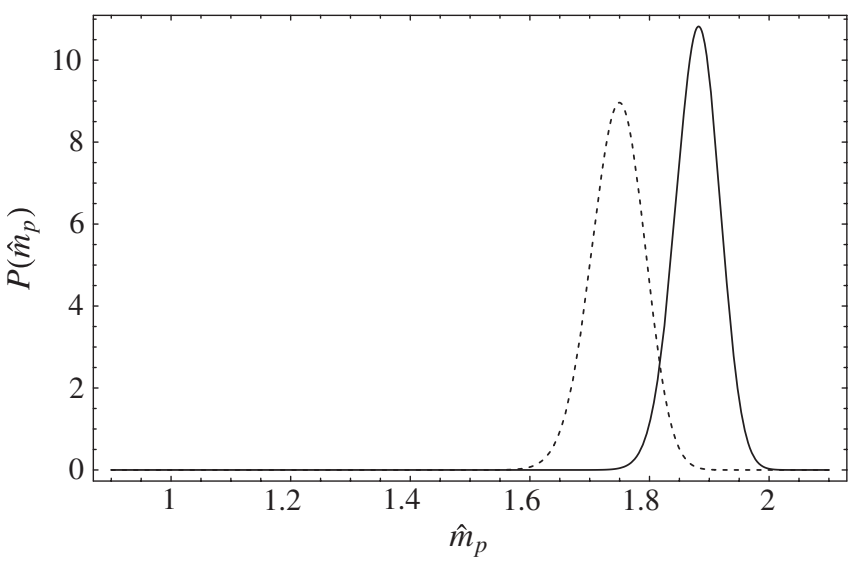

FIG. 3. The normalized distribution $P\left(\hat{m}_{\mathrm{P}}\right)$ for $N=60 \hat{m}_{\mathrm{P}}$ (solid line) and $N=60 \ln \left(\hat{m}_{\mathrm{P}}\right)$ (dashed line) for when the landscape distribution for $m_{\mathrm{P}}$ depends on the inflationary expansion factor. Our universe corresponds to $\hat{m}_{\mathrm{P}}=1$. In both cases $F$ is defined using $\alpha=3$ and $\beta=3 / 2$. Although in both cases selection effects appear to prefer a specific range for $m_{\mathrm{P}}$, our value is far outside of this range. 
assumptions of this appendix, the overwhelming majority of galaxies stem from overdensities that begin with amplitudes many standard deviations away from the norm. Although these galaxies result from relatively unlikely initial overdensities, the fact that they arise within enor- mously larger universes more than compensates for this. This result stems from the sharp dependence on $m_{\mathrm{P}}$ in the inflationary expansion factor. If the proper landscape measure does not contain this factor, then the distribution for $m_{\mathrm{P}}$ would be very different than Fig. 3 indicates.
[1] A. Vilenkin, Phys. Rev. D 27, 2848 (1983); A. D. Linde, Mod. Phys. Lett. A 1, 81 (1986); Phys. Lett. B 175, 395 (1986); A.S. Goncharov, A. D. Linde, and V.F. Mukhanov, Int. J. Mod. Phys. A 2, 561 (1987).

[2] R. Bousso and J. Polchinski, J. High Energy Phys. 06 (2000) 006; L. Susskind, arXiv:hep-th/0302219; M. R. Douglas, J. High Energy Phys. 05 (2003) 046; A. Giryavets, S. Kachru, P. K. Tripathy, and S. P. Trivedi, J. High Energy Phys. 04 (2004) 003; A. Giryavets, S. Kachru, and P. K. Tripathy, J. High Energy Phys. 08 (2004) 002; for a recent review see M. R. Douglas and S. Kachru, Rev. Mod. Phys. 79, 733 (2007); and for dissent see T. Banks, arXiv:hep-th/0412129.

[3] T. Banks, M. Dine, and E. Gorbatov, J. High Energy Phys. 08 (2004) 058

[4] E. Baum, Phys. Lett. B 133, 185 (1983); S. W. Hawking, Phys. Lett. B 134, 403 (1984); S. R. Coleman, Nucl. Phys. B307, 867 (1988); T. Banks, Nucl. Phys. B309, 493 (1988); S. B. Giddings and A. Strominger, Nucl. Phys. B307, 854 (1988).

[5] B. Carter, in Confrontations of Cosmological Theories with Observational Data, I.A.U. Symposium 63, edited by M. Longair (Reidel, Dordrecht, 1974), p. 291; T. Banks, Nucl. Phys. B249, 332 (1985); J. D. Barrow and F. J. Tipler, The Anthropic Cosmological Principle (Oxford University, New York, 1986).

[6] V. Agrawal, S. M. Barr, J. F. Donoghue, and D. Seckel, Phys. Rev. Lett. 80, 1822 (1998); Phys. Rev. D 57, 5480 (1998); B. Feldstein, L. J. Hall, and T. Watari, Phys. Rev. D 74, 095011 (2006).

[7] S. Weinberg, Phys. Rev. Lett. 59, 2607 (1987); H. Martel, P. R. Shapiro, and S. Weinberg, Astrophys. J. 492, 29 (1998).

[8] For earlier work on this idea, see A. D. Linde, Rep. Prog. Phys. 47, 925 (1984); A. D. Sakharov, Zh. Eksp. Teor. Fiz. 87, 375 (1984) [Sov. Phys. JETP 60, 214 (1984)]; Sov. Phys. Usp. 34, 417 (1991); T. Banks, Nucl. Phys. B249, 332 (1985); A. D. Linde, in Three Hundred Years of Gravitation, edited by S.W. Hawking and W. Israel (Cambridge University Press, Cambridge, England, 1987).

[9] M. Tegmark, A. Vilenkin, and L. Pogosian, Phys. Rev. D 71, 103523 (2005).

[10] M. Dine, E. Gorbatov, and S. D. Thomas, arXiv:hep-th/ 0407043; R. Kallosh and A. Linde, J. High Energy Phys. 12 (2004) 004; I. Antoniadis and S. Dimopoulos, Nucl. Phys. B715, 120 (2005); M. Dine, D. O’Neil, and Z. Sun, J. High Energy Phys. 07 (2005) 014; M. Dine, J. High Energy Phys. 01 (2006) 162; M. Dine and Z. Sun, J. High Energy Phys. 01 (2006) 129.

[11] A. Vilenkin, Phys. Rev. Lett. 74, 846 (1995).
[12] M. Tegmark, J. Cosmol. Astropart. Phys. 04 (2005) 001.

[13] A. D. Linde, Phys. Lett. B 160, 243 (1985).

[14] A. D. Linde, Phys. Lett. B 201, 437 (1988); S. Hellerman and J. Walcher, Phys. Rev. D 72, 123520 (2005).

[15] M. Tegmark, A. Aguirre, M. J. Rees, and F. Wilczek, Phys. Rev. D 73, 023505 (2006).

[16] B. J. Carr and M. J. Rees, Nature (London) 278, 605 (1979).

[17] R. Harnik, G. D. Kribs, and G. Perez, Phys. Rev. D 74, 035006 (2006).

[18] L. Clavelli and R. E. White III, arXiv:hep-ph/0609050.

[19] M. Tegmark and M. J. Rees, Astrophys. J. 499, 526 (1998).

[20] M.J. Rees, in Fred Hoyle's Universe, edited by C. Wickramasinghe et al. (Kluwer, Dordrecht, 2003), p. 95; M. L. Graesser, S. D. H. Hsu, A. Jenkins, and M. B. Wise, Phys. Lett. B 600, 15 (2004).

[21] A. Aguirre, Phys. Rev. D 64, 083508 (2001).

[22] A. D. Linde and A. Mezhlumian, Phys. Lett. B 307, 25 (1993); A. D. Linde, D. A. Linde, and A. Mezhlumian, Phys. Lett. B 345, 203 (1995); A. D. Linde and A. Mezhlumian, Phys. Rev. D 53, 4267 (1996).

[23] J. Garcia-Bellido, A. D. Linde, and D. A. Linde, Phys. Rev. D 50, 730 (1994); J. Garcia-Bellido, Nucl. Phys. B423, 221 (1994); J. Garcia-Bellido and A.D. Linde, Phys. Rev. D 52, 6730 (1995).

[24] S. Winitzki and A. Vilenkin, Phys. Rev. D 53, 4298 (1996); A. Vilenkin, Phys. Rev. Lett. 81, 5501 (1998); V. Vanchurin, A. Vilenkin, and S. Winitzki, Phys. Rev. D 61, 083507 (2000); J. Garriga and A. Vilenkin, Phys. Rev. D 64, 023507 (2001); J. Garriga, D. Schwartz-Perlov, A. Vilenkin, and S. Winitzki, J. Cosmol. Astropart. Phys. 01 (2006) 017; V. Vanchurin and A. Vilenkin, Phys. Rev. D 74, 043520 (2006); R. Easther, E. A. Lim, and M. R. Martin, J. Cosmol. Astropart. Phys. 03 (2006) 016; R. Bousso, Phys. Rev. Lett. 97, 191302 (2006); A. Vilenkin, J. Phys. A 40, 6777 (2007); A. Aguirre, S. Gratton, and M. C. Johnson, Phys. Rev. D 75, 123501 (2007).

[25] A. Linde, J. Cosmol. Astropart. Phys. 01 (2007) 022.

[26] N. Arkani-Hamed, S. Dimopoulos, and S. Kachru, arXiv:hep-th/0501082.

[27] A. D. Linde, Phys. Lett. B 238, 160 (1990).

[28] T. Biswas and A. Notari, Phys. Rev. D 74, 043508 (2006).

[29] B. Feldstein, L. J. Hall, and T. Watari, Phys. Rev. D 72, 123506 (2005); J. Garriga and A. Vilenkin, Prog. Theor. Phys. Suppl. 163, 245 (2006).

[30] A. D. Linde, Particle Physics and Inflationary Cosmology (Harwood, Academic, Chur, Switzerland, 1990); D. H. Lyth and A. Riotto, Phys. Rep. 314, 1 (1999).

[31] A. D. Linde, Phys. Lett. B 129, 177 (1983).

[32] A. D. Linde, Phys. Rev. D 49, 748 (1994). 
[33] K. Freese, J. A. Frieman, and A. V. Olinto, Phys. Rev. Lett. 65, 3233 (1990).

[34] N. Arkani-Hamed, P. Creminelli, S. Mukohyama, and M. Zaldarriaga, J. Cosmol. Astropart. Phys. 04 (2004) 001.

[35] D. H. Lyth, J. Cosmol. Astropart. Phys. 11 (2005) 006; M. P. Salem, Phys. Rev. D 72, 123516 (2005).

[36] D. H. Lyth and D. Wands, Phys. Lett. B 524, 5 (2002); T. Moroi and T. Takahashi, Phys. Lett. B 522, 215 (2001); 539, 303(E) (2002); K. Enqvist and M.S. Sloth, Nucl. Phys. B626, 395 (2002).

[37] G. Dvali, A. Gruzinov, and M. Zaldarriaga, Phys. Rev. D 69, 023505 (2004); L. Kofman, arXiv:astro-ph/0303614; G. Dvali, A. Gruzinov, and M. Zaldarriaga, Phys. Rev. D 69, 083505 (2004); C. W. Bauer, M. L. Graesser, and M. P. Salem, Phys. Rev. D 72, 023512 (2005).

[38] M. Dine and A. Kusenko, Rev. Mod. Phys. 76, 1 (2003); M. Trodden, Rev. Mod. Phys. 71, 1463 (1999); A. Strumia, arXiv:hep-ph/0608347.

[39] M. Fukugita and T. Yanagida, Phys. Lett. B 174, 45 (1986); H. Murayama and T. Yanagida, Phys. Lett. B 322, 349 (1994).

[40] V.A. Kuzmin, V. A. Rubakov, and M.E. Shaposhnikov, Phys. Lett. B 155, 36 (1985).

[41] I. Affleck and M. Dine, Nucl. Phys. B249, 361 (1985).

[42] M. Dine, L. Randall, and S. D. Thomas, Nucl. Phys. B458, 291 (1996).

[43] E.W. Kolb and M.S. Turner, The Early Universe (Westview Press, Boulder, CO, 1990).

[44] J. Preskill, M. B. Wise, and F. Wilczek, Phys. Lett. B 120, 127 (1983).

[45] S. Hawking, Mon. Not. R. Astron. Soc. 152, 75 (1971); B. J. Carr and J. E. Lidsey, Phys. Rev. D 48, 543 (1993).

[46] J. C. Niemeyer and K. Jedamzik, Phys. Rev. D 59, 124013 (1999).

[47] V.F. Mukhanov, H. A. Feldman, and R. H. Brandenberger, Phys. Rep. 215, 203 (1992).

[48] H. V. Peiris et al., Astrophys. J. Suppl. Ser. 148, 213 (2003); D. N. Spergel et al., arXiv:astro-ph/0603449.

[49] W. H. Press and P. Schechter, Astrophys. J. 187, 425
(1974); J. M. Bardeen, J. R. Bond, N. Kaiser, and A. S. Szalay, Astrophys. J. 304, 15 (1986).

[50] J. Binney and S. Tremaine, Galactic Dynamics (Princeton University, Princeton, NJ, 1987).

[51] M. J. Rees and J. P. Ostriker, Mon. Not. R. Astron. Soc. 179, 541 (1977).

[52] T. Abel, P. Anninos, Y. Zhang, and M. L. Norman, New Astron. Rev. 2, 181 (1997); P. Anninos, Y. Zhang, T. Abel, and M. L. Norman, New Astron. Rev. 2, 209 (1997).

[53] S. M. Fall and G. Efstathiou, Mon. Not. R. Astron. Soc. 193, 189 (1980).

[54] P. J.E. Peebles, Astrophys. J. 155, 393 (1969); P. J. E. Peebles, Astron. Astrophys. 11, 377 (1971); G. Efstathiou and B. J. T. Jones, Mon. Not. R. Astron. Soc. 186, 133 (1979); M. Steinmetz and M. Bartelmann, Mon. Not. R. Astron. Soc. 272, 570 (1995).

[55] M. J. Rees, Mon. Not. R. Astron. Soc. 176, 483 (1976).

[56] J.P. Cox and R. T. Giuli, Principles of Stellar Structure (Gordon and Breach, New York, 1968).

[57] R. Kippenhahn and A. Weigert, Stellar Structure and Evolution (Springer-Verlag, Berlin, 1994).

[58] D. N. Limber, Astrophys. J. 127, 363 (1958); 127, 387 (1958).

[59] G. Chabrier and I. Baraffe, Astron. Astrophys. 327, 1039 (1997).

[60] S.E. Woosley and T. A. Weaver, Annu. Rev. Astron. Astrophys. 24, 205 (1986); H. A. Bethe, Rev. Mod. Phys. 62, 801 (1990).

[61] K. Nomoto et al., Nucl. Phys. A621, 467c (1997); K. Iwamoto et al., Astrophys. J. Suppl. Ser. 125, 439 (1999).

[62] V.F. Cardone and M. Sereno, Astron. Astrophys. 438, 545 (2005).

[63] A. Linde and V. Mukhanov, J. Cosmol. Astropart. Phys. 04 (2006) 009.

[64] L. J. Hall, T. Watari, and T. T. Yanagida, Phys. Rev. D 73, 103502 (2006).

[65] J. S. Bullock, A. Dekel, T. S. Kolatt, A. V. Kravtsov, A. A. Klypin, C. Porciani, and J. R. Primack, Astrophys. J. 555, 240 (2001). 